\title{
A DISCRETE CONTACT MODEL FOR CROWD MOTION
}

\author{
Bertrand Maury ${ }^{1}$ And Juliette Venel $^{1}$
}

\begin{abstract}
The aim of this paper is to develop a crowd motion model designed to handle highly packed situations. The model we propose rests on two principles: we first define a spontaneous velocity which corresponds to the velocity each individual would like to have in the absence of other people. The actual velocity is then computed as the projection of the spontaneous velocity onto the set of admissible velocities (i.e. velocities which do not violate the non-overlapping constraint). We describe here the underlying mathematical framework, and we explain how recent results by J.F. Edmond and L. Thibault on the sweeping process by uniformly prox-regular sets can be adapted to handle this situation in terms of well-posedness. We propose a numerical scheme for this contact dynamics model, based on a prediction-correction algorithm. Numerical illustrations are finally presented and discussed.
\end{abstract}

Mathematics Subject Classification. 34A60, 47H04, 70F35, 90C46.

Received December 19, 2008. Revised December 18, 2009.

Published online June 24, 2010.

\section{INTRODUCTION}

Walking behaviour of pedestrians has given rise to a large amount of empirical studies over the last decades. Qualitative data (preferences, walk tendencies) have been collected by Fruin [18], Navin and Wheeler [41], Henderson [23] and, more recently, by Weidmann [48]. From these observations, several strategies for crowd motion modelling have been proposed, and can be classified with respect to the way they handle people density (Lagrangian description of individuals or macroscopic approach), and to the nature of motion phenomena (deterministic or stochastic). Among discrete and stochastic models, let us mention Cellular Automata [3,8,40, 42], models based on networks [19] as route choice models [4,5] and queuing models [34,49]. In these models, each cell or node is either empty or occupied by a single person and people's motion always satisfies this rule. In cellular automata models, there are two manners of moving people during a time step. With the first one, positions are updated one by one with a random order (Random Sequential Update). The second method consists of updating simultaneously all positions (Parallel Update). If several people want to reach the same cell, only one of them (randomly chosen) is allowed to move. In route choice models, people move on a network. Each model is based on a route choice set. Most choice set generation procedures are based on shortest route search and use shortest paths algorithms. Queuing models use Markov-chain models to describe how pedestrians move from one node of the network to another.

In [21], a microscopic model called social force model is presented. It describes crowd motion with a system of differential equations. The acceleration of an individual is obtained according to Newton's law. Several forces

Keywords and phrases. Crowd motion model, contact dynamics, convex analysis, differential inclusion, prox-regularity.

${ }^{1}$ Laboratoire de Mathématiques, Université Paris-Sud XI, 91405 Orsay Cedex, France. juliette.venel@math.u-psud.fr 
are introduced as for example a term describing the acceleration towards the desired velocity or a repulsion force reflecting that a pedestrian tends to keep a certain distance from other people and obstacles. Moreover macroscopic models have been proposed. In [23], pedestrian traffic dynamics is firstly compared with fluid dynamics. Some models $[20,23,24]$ are based on gas-kinetic theory. Other models [25-28] rest on a set of partial differential equations describing the conservation of flow equation. In [1,15], second order models based on hyperbolic conservation laws are introduced, where a phenomenological relation describes how the crowd modifies its own speed. In [9,37], crowd motion modelling rests on optimal transportation principles.

Several softwares have been developed: PedGo [31], SimPed [13], Legion [44], Mipsim [24] or Exodus [19]. Some commonly observed collective patterns are now considered as standard benchmarks for those numerical simulations. Among these phenomena of self-organization, there is the formation of lanes formed naturally by people moving in opposite directions. In this way, strong interactions with oncoming pedestrians are reduced, and a higher walking speed is possible. Another phenomena is the formation of arches upstream the exit during the evacuation of a room. These patterns are recovered by CA-models $[30,43]$ and by the social force model $[21,22]$.

The case of evacuation in emergency situations is of particular importance in terms of applications (observance of security rules, computer-assisted design of public buildings, appropriate positioning of exit signs). Numerical simulations may allow to estimate evacuation time (to be compared for example with the duration of fire propagation) and also to predict areas where high density will appear. As pointed out by Helbing et al. [22], emergency situations do not fit into the standard framework of pedestrian traffic flow. When people stroll around without hurry, they tend to keep a certain distance from each other and from obstacles. In an emergency situation, the motion of individuals is governed by different rules. In particular, the contact with walls or other people is no longer avoided. Some strategies have been proposed to adapt social walk models to highly congested situation (see again [22]).

We propose here an approach which relies on the very consideration that actual motion in emergency situations is governed by the opposition between achievement of individual satisfaction (people struggle to escape as quickly as possible, regardless of the global efficiency) and congestion. In particular, we aim at integrating the direct conflict between people in the model, in order to estimate in some way interaction forces between them, and therefore provide a way to estimate the local risk of casualties. The model introduced here is a microscopic, deterministic and first order model.

The microscopic model we propose rests on two principles. On the one hand, each individual has a spontaneous velocity (or desired velocity) that he would like to have in the absence of other people. On the other hand, the actual velocity must take into account congestion. Those two principles lead us to define the actual velocity field as the projection of the spontaneous velocity onto the set of admissible velocities (regarding the non-overlapping constraints). The flexibility of this model lies in its first point: every choice of spontaneous velocity can be made and so every existing model for predicting crowd motion can be integrated here. The key feature of the model is the second point which concerns handling of contacts. The model we propose is essentially designed to handle actual contacts between individuals, which are likely to happen in highly packed situations only. Sophisticated strategies can be included in the definition of the spontaneous (or desired) velocity, and this velocity can be made dependent, e.g., on the distance to the preceding pedestrian, in the spirit of traffic flows. In particular, such models could be based on a "comfort distance" between people (distance below which people do not feel good), and this distance might depend on cultural aspects, and possibly some compromise between comfort and self estimation of the emergency character of the situation. Those models can be included in the definition of the spontaneous velocity. Yet, as the paper is dedicated to the very difficulties raised by the non-smooth handling of the non-overlapping constraint, we shall restrict here to a very basic definition of the spontaneous velocity (see [46] for more sophisticated models).

This model allows us to deal with a large number of pedestrians without constraints on the time-step. Moreover, in this model, the handling of contacts relies on Lagrange multipliers, each of which can be interpreted as an interaction pressure between two individuals in contact. Thus the model may predict the zones in which undergone pressure (and consequently discomfort or even casualties) is the highest. 


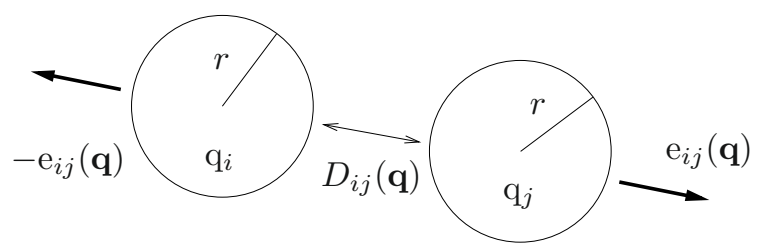

FiguRE 1. Notations.

By specifying the link between the actual and spontaneous velocities, the evolution problem takes the form of a first order differential inclusion. This type of evolution problem has been extensively studied in the 1970's, with the theory of maximal monotone operators (see e.g. [7]). A few years later, Moreau considered similar problems with time-dependent multivalued operator, namely sweeping processes by convex sets (see [39]). Since then, important improvements have been developed by weakening the convexity assumption with the concept of prox-regularity. The well-posedness of our evolution problem can be established by means of recent results of Edmond and Thibault [16] concerning sweeping processes by uniformly prox-regular sets.

The paper is structured as follows: In Section 2, we present the model and establish its well-posedness. In Section 3, we propose a numerical scheme, and detail the overall solution method. Section 4 is devoted to some illustrations of the numerical algorithm.

\section{Modelling}

We consider $N$ persons identified to rigid disks. For convenience, the disks are supposed here to have the same radius $r$. The centre of the $i$ th disk is denoted by $\mathrm{q}_{i}$ (see Fig. 1). Since overlapping is forbidden, the vector of positions $\mathbf{q}=\left(\mathrm{q}_{1}, \ldots, \mathrm{q}_{N}\right) \in \mathbb{R}^{2 N}$ (equipped with the Euclidean norm) is required to belong to the following set:

Definition 1.1 (set of feasible configurations).

$$
Q=\left\{\mathbf{q} \in \mathbb{R}^{2 N}, D_{i j}(\mathbf{q}) \geq 0 \quad \forall i<j\right\}
$$

where $D_{i j}(\mathbf{q})=\left|\mathrm{q}_{i}-\mathrm{q}_{j}\right|-2 r$ is the signed distance between disks $i$ and $j$.

We consider as given the vector of spontaneous velocities denoted by

$$
\mathbf{U}(\mathbf{q})=\left(\mathbf{U}_{1}(\mathbf{q}), \ldots, \mathbf{U}_{N}(\mathbf{q})\right) \in \mathbb{R}^{2 N}
$$

$\mathbf{U}_{i}$ is the spontaneous velocity of individual $i$, which may depend on its own position $\left(\mathbf{U}_{i}=\mathbf{U}_{i}\left(\mathbf{q}_{i}\right)\right.$, see Sect. 4 for examples of such a situation), but also on other people's positions, that is why we keep here $\mathbf{U}_{i}=\mathbf{U}_{i}(\mathbf{q})$. To define the actual velocity, we introduce the following set:

Definition 1.2 (set of feasible velocities).

$$
\mathcal{C}_{\mathbf{q}}=\left\{\mathbf{v} \in \mathbb{R}^{2 N}, \forall i<j \quad D_{i j}(\mathbf{q})=0 \Rightarrow \mathbf{G}_{i j}(\mathbf{q}) \cdot \mathbf{v} \geq 0\right\}
$$

with

$$
\mathbf{G}_{i j}(\mathbf{q})=\nabla D_{i j}(\mathbf{q})=\left(0, \ldots, 0,-\mathrm{e}_{i j}(\mathbf{q}), 0, \ldots, 0, \mathrm{e}_{i j}(\mathbf{q}), 0, \ldots, 0\right) \in \mathbb{R}^{2 N} \text { and } \mathrm{e}_{i j}(\mathbf{q})=\frac{\mathrm{q}_{j}-\mathrm{q}_{i}}{\left|\mathrm{q}_{j}-\mathrm{q}_{i}\right|}
$$




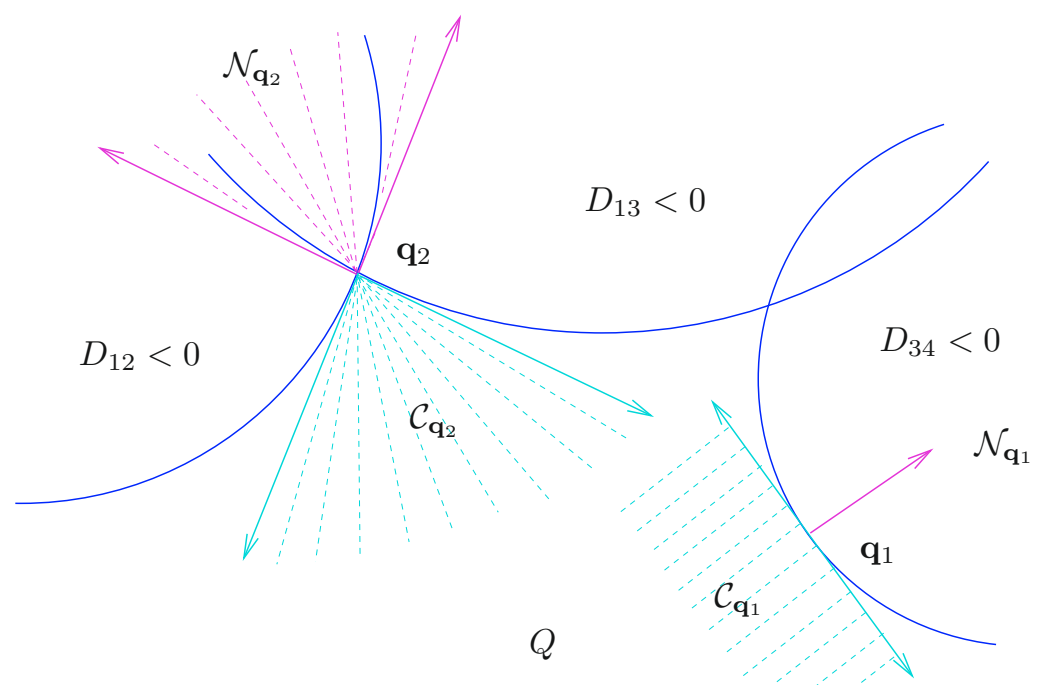

Figure 2. Cones $\mathcal{C}_{\mathbf{q}}$ and $\mathcal{N}_{\mathbf{q}}$.

The actual velocity field is defined as the feasible field which is the closest to $\mathbf{U}$ in the least square sense, which writes

$$
\left\{\begin{array}{l}
\frac{\mathrm{d} \mathbf{q}}{\mathrm{d} t}=\mathrm{P}_{\mathcal{C}_{\mathbf{q}}} \mathbf{U}(\mathbf{q}), \\
\mathbf{q}(0)=\mathbf{q}_{0} \in Q,
\end{array}\right.
$$

where $\mathrm{P}_{\mathcal{C}_{\mathbf{q}}}$ denotes the Euclidean projection onto the closed convex cone $\mathcal{C}_{\mathbf{q}}$.

Remark 1.3. Despite its formal simplicity, this model does not fit directly into a standard framework. Indeed the set $\mathcal{C}_{\mathbf{q}}$ does not continuously depend on $\mathbf{q}$. If no contact holds, the velocity is not constrained and $\mathcal{C}_{\mathbf{q}}=\mathbb{R}^{2 N}$. With a single contact, the set $\mathcal{C}_{\mathbf{q}}$ becomes a half-space.

\section{Mathematical FRAMEWORK}

\subsection{Reformulation}

Let us reformulate the problem by introducing $\mathcal{N}_{\mathbf{q}}$, the outward normal cone to the set of feasible configurations $Q$, which is defined as the polar cone of $\mathcal{C}_{\mathbf{q}}$.

Definition 2.1 (outward normal cone).

$$
\mathcal{N}_{\mathbf{q}}=\mathcal{C}_{\mathbf{q}}^{\circ}=\left\{\mathbf{w} \in \mathbb{R}^{2 N}, \mathbf{w} \cdot \mathbf{v} \leq 0 \quad \forall \mathbf{v} \in \mathcal{C}_{\mathbf{q}}\right\} .
$$

Remark 2.2. In Figure 2, we represent the set $Q \subset \mathbb{R}^{2 N}$ which is defined as an intersection of convex sets' complements. In the case of a single contact (configuration $\mathbf{q}_{1}$ ), we remark that the cone $\mathcal{N}_{\mathbf{q}_{1}}$ is generated by the vector $-\mathbf{G}_{34}\left(\mathbf{q}_{1}\right)$ that is up to a constant, the outward normal vector to the domain $D_{34} \geq 0$. In the case of two or more contacts, the configuration $\mathbf{q}_{2}$ does not belong to a smooth surface and the cone $\mathcal{N}_{\mathbf{q}_{2}}$ (generated by $-\mathbf{G}_{12}\left(\mathbf{q}_{2}\right)$ and $\left.-\mathbf{G}_{13}\left(\mathbf{q}_{2}\right)\right)$ generalizes somehow the notion of the outward normal direction.

Thanks to Farkas' Lemma (see [10]), the outward normal cone can be expressed

$$
\mathcal{N}_{\mathbf{q}}=\left\{-\sum \lambda_{i j} \mathbf{G}_{i j}(\mathbf{q}), \lambda_{i j} \geq 0, D_{i j}(\mathbf{q})>0 \Longrightarrow \lambda_{i j}=0\right\} .
$$




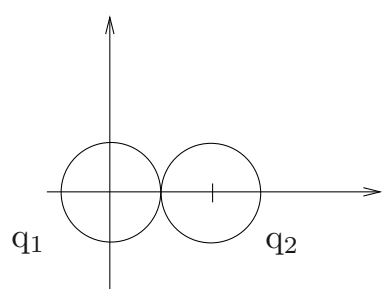

configuration $\mathbf{q}$

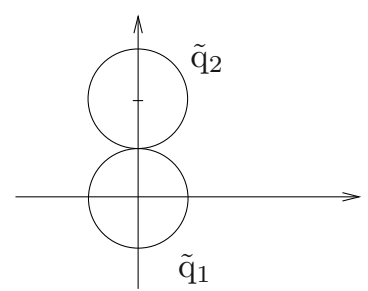

configuration $\widetilde{\mathbf{q}}$

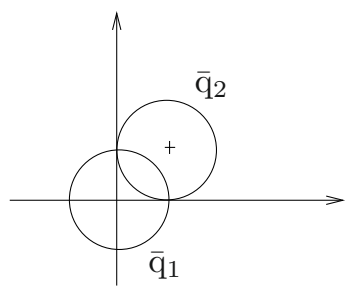

configuration $\overline{\mathbf{q}}=\frac{\mathbf{q}+\widetilde{\mathbf{q}}}{2}$

FiguRE 3. Lack of convexity.

Let us recall the classical orthogonal decomposition of a Hilbert space as the sum of mutually polar cones (see [38]):

Using this property, we get:

$$
\mathrm{P}_{\mathcal{C}_{\mathbf{q}}}+\mathrm{P}_{\mathcal{N}_{\mathbf{q}}}=\mathrm{Id}
$$

$$
\frac{\mathrm{d} \mathbf{q}}{\mathrm{d} t}=\mathrm{P}_{\mathcal{C}_{\mathbf{q}}} \mathbf{U}(\mathbf{q})=\mathbf{U}(\mathbf{q})-\mathrm{P}_{\mathcal{N}_{\mathbf{q}}} \mathbf{U}(\mathbf{q})
$$

Since $\mathrm{P}_{\mathcal{N}_{\mathbf{q}}} \mathbf{U}(\mathbf{q}) \in \mathcal{N}_{\mathbf{q}}$, we obtain a new formulation for (1.1)

$$
\left\{\begin{array}{l}
\frac{\mathrm{d} \mathbf{q}}{\mathrm{d} t}+\mathcal{N}_{\mathbf{q}} \ni \mathbf{U}(\mathbf{q}) \\
\mathbf{q}(0)=\mathbf{q}_{0}
\end{array}\right.
$$

The problem reads as a first order differential inclusion involving the multivalued operator $\mathcal{N}$.

Remark 2.3. In the absence of contacts in the configuration $\mathbf{q}$, the set of feasible velocities $\mathcal{C}_{\mathbf{q}}$ is equal to the whole space $\mathbb{R}^{2 N}$, and consequently the outward normal cone $\mathcal{N}_{\mathbf{q}}$ is reduced to $\{0\}$. In that case, the first relation of (2.4) states that the actual velocity equals to the spontaneous velocity:

$$
\frac{\mathrm{d} \mathbf{q}}{\mathrm{d} t}=\mathbf{U}(\mathbf{q})
$$

If any contact exists, the differential inclusion means that the configuration $\mathbf{q}$, submitted to $\mathbf{U}(\mathbf{q})$, has to evolve while remaining in $Q$.

Let us first study a special situation where standard theory can be applied. Consider $N$ individuals in a corridor. In that case, as people cannot leap across each other, it is natural to restrict the set of feasible configurations to one of its connected components:

$$
Q=\left\{\mathbf{q}=\left(\mathrm{q}_{1}, \ldots, \mathrm{q}_{N}\right) \in \mathbb{R}^{N}, \mathrm{q}_{i+1}-\mathrm{q}_{i} \geq 2 r\right\}
$$

In this very situation, as $Q$ is closed and convex, the multivalued operator $\mathbf{q} \longmapsto \mathcal{N}_{\mathbf{q}}$ identifies to the subdifferential of the indicatrix function of $Q$ :

$$
\partial I_{Q}(\mathbf{q})=\left\{\mathbf{v}, I_{Q}(\mathbf{q})+(\mathbf{v}, \mathbf{h}) \leq I_{Q}(\mathbf{q}+\mathbf{h}) \quad \forall \mathbf{h}\right\}, I_{Q}(\mathbf{q})=\mid \begin{array}{rll}
0 & \text { if } & \mathbf{q} \in Q \\
+\infty & \text { if } \quad \mathbf{q} \notin Q
\end{array}
$$

therefore $\mathbf{q} \longmapsto \mathcal{N}_{\mathbf{q}}$ is maximal monotone. In that case, as soon as the spontaneous velocity is regular (say Lipschitz), standard theory (see e.g. Brezis [7]) ensures well-posedness. Yet, as illustrated in Figure 3, $Q$ is not convex in general and the operator $\mathbf{q} \longmapsto \mathcal{N}_{\mathbf{q}}$ is not monotone. So we cannot apply the same arguments as 


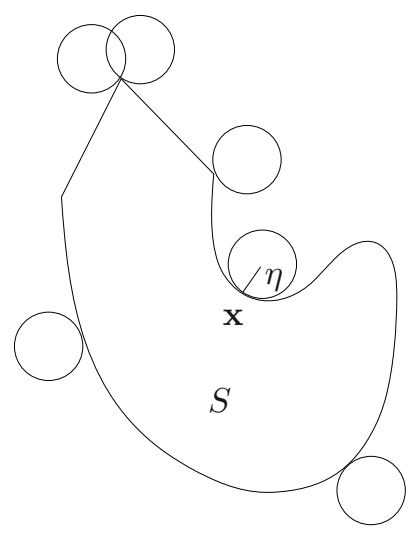

FigURE 4. $\eta$-prox-regular set.

in the case of a straight motion. By lack of convexity, the projection onto $Q$ is not everywhere well-defined. However the set $Q$ satisfies a weaker property in the sense that the projection onto $Q$ is still well-defined in its neighbourhood. Indeed, $Q$ is uniformly prox-regular, which is the suitable property to ensure well-posedness. Let us give some definitions to specify the general mathematical framework.

Definition 2.4. Let $S$ be a closed subset of a Hilbert space $H$.

We define the proximal normal cone to $S$ at $\mathbf{x}$ by:

$$
\mathrm{N}(S, \mathbf{x})=\left\{\mathbf{v} \in H, \exists \alpha>0, \mathbf{x} \in \mathrm{P}_{S}(\mathbf{x}+\alpha \mathbf{v})\right\},
$$

where

$$
\mathrm{P}_{S}(\mathbf{y})=\left\{\mathbf{z} \in S, d_{S}(\mathbf{y})=|\mathbf{y}-\mathbf{z}|\right\}, \text { with } d_{S}(\mathbf{y})=\inf _{\mathbf{z} \in S}|\mathbf{y}-\mathbf{z}| .
$$

Following [11], we define the concept of uniform prox-regularity as follows:

Definition 2.5. Let $S$ be a closed subset of a Hilbert space H.S is said $\eta$-prox-regular if for all $\mathbf{x} \in \partial S$ and $\mathbf{v} \in \mathrm{N}(S, \mathbf{x}),|\mathbf{v}|=1$ we have:

$$
B(\mathbf{x}+\eta \mathbf{v}, \eta) \cap S=\emptyset .
$$

Equivalently, $S$ is $\eta$-prox-regular if for all $\mathbf{y} \in S, \mathbf{x} \in \partial S$ and $\mathbf{v} \in \mathrm{N}(S, \mathbf{x})$,

$$
\mathbf{v} \cdot(\mathbf{y}-\mathbf{x}) \leq \frac{|\mathbf{v}|}{2 \eta}|\mathbf{y}-\mathbf{x}|^{2} .
$$

In an Euclidean space, $S$ is $\eta$-prox-regular if an external tangent ball with radius smaller than $\eta$ can be rolled around it (see Fig. 4). Moreover, this definition ensures that the projection onto such a set is well-defined in its neighbourhood. The following remark will be useful later.

Remark 2.6. If there exists $\alpha>0$ satisfying $\mathbf{x} \in \mathrm{P}_{S}(\mathbf{x}+\alpha \mathbf{v})$ then

$$
\forall \beta \geq 0, \beta \leq \alpha, \mathbf{x} \in \mathrm{P}_{S}(\mathbf{x}+\beta \mathbf{v}) .
$$

Definition 2.7. The proximal subdifferential of function $d_{S}$ at $\mathrm{x}$ is the set

$$
\partial^{P} d_{S}(\mathbf{x})=\left\{\mathbf{v} \in H, \exists M, \alpha>0, d_{S}(\mathbf{y})-d_{S}(\mathbf{x})+M|\mathbf{y}-\mathbf{x}|^{2} \geq \mathbf{v} \cdot(\mathbf{y}-\mathbf{x}), \forall \mathbf{y} \in B(\mathbf{x}, \alpha)\right\}
$$


Let us specify the link between the previous subdifferential and the proximal normal cone, which is proved in $[6,12]$.

Proposition 2.8. The following relation holds true:

$$
\partial^{P} d_{S}(\mathbf{x})=\mathrm{N}(S, \mathbf{x}) \cap \overline{B(0,1)}
$$

Remark 2.9. A set $C \subset H$ is convex if and only if it is $\infty$-prox-regular. In this case $\mathrm{N}(C, \mathbf{x})=\partial I_{C}(\mathbf{x})$ for all $\mathbf{x} \in C$.

We now come to the main result of this section.

Theorem 2.10. Assume that $\mathbf{U}$ is Lipschitz and bounded. Then, for all $T>0$ and all $\mathbf{q}_{0} \in Q$, the following problem

$$
\left\{\begin{array}{l}
\frac{\mathrm{d} \mathbf{q}}{\mathrm{d} t}+\mathcal{N}_{\mathbf{q}} \ni \mathbf{U}(\mathbf{q}) \\
\mathbf{q}(0)=\mathbf{q}_{0},
\end{array}\right.
$$

has one and only one absolutely continuous solution $\mathbf{q}(\cdot)$ over $[0, T]$.

This well-posedness can be obtained by using results in [16,17] as soon as we prove that $Q$ is uniformly prox-regular and that the set $\mathcal{N}_{\mathbf{q}}$ identifies to the proximal normal cone to $Q$ at $\mathbf{q}$. This is the core of next subsection.

Remark 2.11. It can be shown that the solution given by Theorem 2.10 satisfies the initial differential equation (2.3) (see [2]).

\subsection{Prox-regularity of $Q$}

Let us consider the set

$$
Q_{i j}=\left\{\mathbf{q} \in \mathbb{R}^{2 N}, D_{i j}(\mathbf{q}) \geq 0\right\} .
$$

Proposition 2.12. Let $S$ be a closed subset of $\mathbb{R}^{n}$ whose boundary $\partial S$ is an oriented $C^{2}$ hypersurface. For each $\mathbf{x} \in \partial S$, we denote by $\nu(\mathbf{x})$ the outward normal to $S$ at $\mathbf{x}$. Then, for each $\mathbf{x} \in \partial S$, the proximal normal cone to $S$ at $\mathbf{x}$ is generated by $\nu(\mathbf{x})$, i.e.

$$
\mathrm{N}(S, \mathbf{x})=\mathbb{R}^{+} \nu(\mathbf{x})
$$

Proof. The proof is a straightforward computation (see Prop. 3.2 in [45]).

We can also deduce the expression of the proximal normal cone to $Q_{i j}$.

Corollary 2.13. For all $\mathbf{q} \in \partial Q_{i j}$,

$$
\mathrm{N}\left(Q_{i j}, \mathbf{q}\right)=-\mathbb{R}^{+} \mathbf{G}_{i j}(\mathbf{q})
$$

By Definition 2.5, the constant of prox-regularity equals to the largest radius of a "rolling external ball". In order to estimate its radius, tools of differential geometry can be used. More precisely, to show that the set $Q_{i j}$ is uniformly prox-regular, we can apply the following theorem, that is proved in [14].

Theorem 2.14. Let $C$ be a closed convex subset of $\mathbb{R}^{n}$ such that $\partial C$ is an oriented $C^{2}$ hypersurface of $\mathbb{R}^{n}$. We denote by $\nu_{C}(\mathbf{x})$ the outward normal to $C$ at $\mathbf{x}$ and by $\rho_{1}(\mathbf{x}), \ldots, \rho_{n-1}(\mathbf{x}) \geq 0$ the principal curvatures of $C$ at $\mathbf{x}$. We suppose that

$$
\rho=\sup _{\mathbf{x} \in \partial C} \sup _{1 \leq i \leq n-1} \rho_{i}(\mathbf{x})<\infty .
$$

Then $S=\mathbb{R}^{n} \backslash \operatorname{int}(C)$ is a $\eta$-prox-regular set with $\eta=\frac{1}{\rho}$. 
Proposition 2.15. $Q_{i j}$ is $\eta_{0}$-prox-regular with $\eta_{0}=r \sqrt{2}$.

Proof. The set $\operatorname{int}\left(Q_{i j}\right)$ is obviously the complement of a convex set $C$ which satisfies the assumptions of Theorem 2.14. The constant of prox-regularity of $Q_{i j}$ can be obtained by calculating its principal curvatures, which are the eigenvalues of Weingarten endomorphism. Let $\mathbf{q} \in \partial Q_{i j}$, the outward normal to $C$ at $\mathbf{q}$ is equal to $-\nu(\mathbf{q})$, where

$$
\nu(\mathbf{q})=-\frac{\mathbf{G}_{i j}(\mathbf{q})}{\sqrt{2}}=\frac{\left(0, \ldots, 0, \mathrm{e}_{i j}(\mathbf{q}), 0, \ldots, 0,-\mathrm{e}_{i j}(\mathbf{q}), 0, \ldots, 0\right)}{\sqrt{2}} .
$$

Weingarten endomorphism is written as follows, for all tangent vectors $\mathbf{h} \in T_{\mathbf{q}}\left(\partial Q_{i j}\right)$,

$$
\mathbf{W}_{\mathbf{q}}(\mathbf{h}):=-\mathrm{D} \nu(\mathbf{q})[\mathbf{h}]=\frac{1}{\sqrt{2}\left|\mathrm{q}_{j}-\mathrm{q}_{i}\right|}\left(0, \ldots, 0,-\mathrm{P}_{\mathrm{e}_{i j}^{\perp}}\left(\mathrm{h}_{j}-\mathrm{h}_{i}\right), 0, \ldots, 0, \mathrm{P}_{\mathrm{e}_{i j}^{\perp}}\left(\mathrm{h}_{j}-\mathrm{h}_{i}\right), 0, \ldots, 0\right),
$$

with

$$
\mathrm{P}_{\mathrm{e}_{i j}^{\perp}}\left(\mathrm{h}_{j}-\mathrm{h}_{i}\right)=\left(\mathrm{h}_{j}-\mathrm{h}_{i}\right)-\left[\left(\mathrm{h}_{j}-\mathrm{h}_{i}\right) \cdot \mathrm{e}_{i j}\right] \mathrm{e}_{i j} .
$$

After some computations, we deduce that the endomorphism $\mathbf{W}_{\mathbf{q}}$ has two eigenvalues, 0 and $\sqrt{2} /\left|\mathrm{q}_{j}-\mathrm{q}_{i}\right|$, and the latter is equal to $1 /(r \sqrt{2})$, which ends the proof.

Now let us study the set of feasible configurations $Q$, that is the intersection of all sets $Q_{i j}$. We begin to determine its proximal normal cone.

Proposition 2.16. For all $\mathbf{q} \in Q, \mathrm{~N}(Q, \mathbf{q})=\sum \mathrm{N}\left(Q_{i j}, \mathbf{q}\right)=\mathcal{N}_{\mathbf{q}}$.

Proof. The second equality follows from (2.1) and Corollary 2.13. Let us prove the first one. If $\mathbf{q} \in \operatorname{int}(Q)$, then for each couple $(i, j), \mathbf{q} \in \operatorname{int}\left(Q_{i j}\right)$, which implies

$$
\mathrm{N}(Q, \mathbf{q})=\{0\}=\sum \mathrm{N}\left(Q_{i j}, \mathbf{q}\right) .
$$

We now consider $\mathbf{q} \in \partial Q$ and introduce the following set:

$$
I_{\text {contact }}=\left\{(i, j), i<j, D_{i j}(\mathbf{q})=0\right\}=\left\{(i, j), i<j, \mathbf{q} \in \partial Q_{i j}\right\} .
$$

First, we check that $\mathrm{N}\left(Q_{i j}, \mathbf{q}\right) \subset \mathrm{N}(Q, \mathbf{q})$. Let $(i, j)$ belong to $I_{\text {contact }}$ (otherwise the previous inclusion is obvious), we consider $\mathbf{w} \in \mathrm{N}\left(Q_{i j}, \mathbf{q}\right) \backslash\{0\}$ and we set $\mathbf{v}=\mathbf{w} /|\mathbf{w}|$. By Proposition 2.8, $\mathbf{v} \in \partial^{P} d_{Q_{i j}}(\mathbf{q})$ and thus

$$
\exists M, \alpha>0, d_{Q_{i j}}(\tilde{\mathbf{q}})-d_{Q_{i j}}(\mathbf{q})+M|\tilde{\mathbf{q}}-\mathbf{q}|^{2} \geq \mathbf{v} \cdot(\tilde{\mathbf{q}}-\mathbf{q}), \forall \tilde{\mathbf{q}} \in B(\mathbf{q}, \alpha) .
$$

Since $d_{Q_{i j}}(\mathbf{q})=0=d_{Q}(\mathbf{q})$ and $d_{Q_{i j}}(\tilde{\mathbf{q}}) \leq d_{Q}(\tilde{\mathbf{q}})$, it follows that

$$
\exists M, \alpha>0, d_{Q}(\tilde{\mathbf{q}})-d_{Q}(\mathbf{q})+M|\tilde{\mathbf{q}}-\mathbf{q}|^{2} \geq \mathbf{v} \cdot(\tilde{\mathbf{q}}-\mathbf{q}), \forall \tilde{\mathbf{q}} \in B(\mathbf{q}, \alpha) .
$$

Therefore $\mathbf{v} \in \partial^{P} d_{Q}(\mathbf{q})$ and $\mathbf{w} \in \mathrm{N}(Q, \mathbf{q})$. Consequently, for each couple $(i, j) \in I_{\text {contact }}$, we obtain $\mathrm{N}\left(Q_{i j}, \mathbf{q}\right) \subset \mathrm{N}(Q, \mathbf{q})$ as required. We now want to prove

$$
\sum \mathrm{N}\left(Q_{i j}, \mathbf{q}\right) \subset \mathrm{N}(Q, \mathbf{q}) .
$$

It suffices to show that

$$
\forall \mathbf{w}_{1}, \mathbf{w}_{2} \in \mathrm{N}(Q, \mathbf{q}) \backslash\{0\}, \mathbf{w}=\mathbf{w}_{1}+\mathbf{w}_{2} \in \mathrm{N}(Q, \mathbf{q}) .
$$

Let $\mathbf{w}_{1}$ and $\mathbf{w}_{2}$ belong to $\mathrm{N}(Q, \mathbf{q}) \backslash\{0\}$, we set $\mathbf{w}=\mathbf{w}_{1}+\mathbf{w}_{2}, \mathbf{v}_{1}=\mathbf{w}_{1} /\left|\mathbf{w}_{1}\right|$ and $\mathbf{v}_{2}=\mathbf{w}_{2} /\left|\mathbf{w}_{2}\right|$. By Proposition 2.8, there exist $M_{1}, M_{2} \geq 0, \alpha_{1}, \alpha_{2}>0$ such that

$$
\begin{aligned}
& d_{Q}(\tilde{\mathbf{q}})-d_{Q}(\mathbf{q})+M_{1}|\tilde{\mathbf{q}}-\mathbf{q}|^{2} \geq \mathbf{v}_{1} \cdot(\tilde{\mathbf{q}}-\mathbf{q}), \quad \forall \tilde{\mathbf{q}} \in B\left(\mathbf{q}, \alpha_{1}\right), \\
& d_{Q}(\tilde{\mathbf{q}})-d_{Q}(\mathbf{q})+M_{2}|\tilde{\mathbf{q}}-\mathbf{q}|^{2} \geq \mathbf{v}_{2} \cdot(\tilde{\mathbf{q}}-\mathbf{q}), \quad \forall \tilde{\mathbf{q}} \in B\left(\mathbf{q}, \alpha_{2}\right) .
\end{aligned}
$$


So $\mathbf{w}=\left|\mathbf{w}_{1}\right| \mathbf{v}_{1}+\left|\mathbf{w}_{2}\right| \mathbf{v}_{2}$ and the vector $\mathbf{v}=\mathbf{w} /\left(\left|\mathbf{w}_{1}\right|+\left|\mathbf{w}_{2}\right|\right)$ satisfies $|\mathbf{v}| \leq 1$. Furthermore $\mathbf{v}=t \mathbf{v}_{1}+(1-t) \mathbf{v}_{2}$, where

$$
t=\frac{\left|\mathbf{w}_{1}\right|}{\left|\mathbf{w}_{1}\right|+\left|\mathbf{w}_{2}\right|}
$$

For $\alpha=\min \left(\alpha_{1}, \alpha_{2}\right)$ and $M=t M_{1}+(1-t) M_{2}$, the following relation holds

$$
d_{Q}(\tilde{\mathbf{q}})-d_{Q}(\mathbf{q})+M|\tilde{\mathbf{q}}-\mathbf{q}|^{2} \geq \mathbf{v} \cdot(\tilde{\mathbf{q}}-\mathbf{q}), \forall \tilde{\mathbf{q}} \in B(\mathbf{q}, \alpha)
$$

Hence $\mathbf{v} \in \partial^{P} d_{Q}(\mathbf{q})$ and $\mathbf{w} \in \mathrm{N}(Q, \mathbf{q})$. To conclude, it remains to check that

$$
\mathrm{N}(Q, \mathbf{q}) \subset \sum \mathrm{N}\left(Q_{i j}, \mathbf{q}\right)
$$

By $(2.2)$, any $\mathbf{w} \in \mathrm{N}(Q, \mathbf{q})$ can be written $\mathbf{w}=\mathbf{v}+\mathbf{z}=\mathrm{P}_{\mathcal{N}_{\mathbf{q}}} \mathbf{w}+\mathrm{P}_{\mathcal{C}_{\mathbf{q}}} \mathbf{w}$, with $\mathbf{v} \perp \mathbf{z}$. Suppose $\mathbf{z} \neq 0$. Since $\mathbf{w} \in \mathrm{N}(Q, \mathbf{q})$, there exists $t>0$ such that $\mathbf{q} \in \mathrm{P}_{Q}(\mathbf{q}+t \mathbf{w})$. Let

$$
s=\min (t, \epsilon) \text { with } \epsilon=\min _{(i, j) \notin I_{\text {contact }}} \frac{D_{i j}(\mathbf{q})}{\sqrt{2}|\mathbf{z}|},
$$

by Remark 2.6, we know that $\mathbf{q} \in \mathrm{P}_{Q}(\mathbf{q}+s \mathbf{w})$. Now set

$$
\tilde{\mathbf{q}}=\mathbf{q}+s \mathbf{w}-s \mathbf{v}=\mathbf{q}+s \mathbf{z}
$$

and show that $\tilde{\mathbf{q}} \in Q$. By convexity of $D_{i j}$, we have

$$
D_{i j}(\tilde{\mathbf{q}}) \geq D_{i j}(\mathbf{q})+s \mathbf{G}_{i j}(\mathbf{q}) \cdot \mathbf{z}, \forall(i, j) .
$$

In addition, for $(i, j) \in I_{\text {contact }}$, it yields $\mathbf{G}_{i j}(\mathbf{q}) \cdot \mathbf{z} \geq 0$, because $\mathbf{z} \in \mathcal{C}_{\mathbf{q}}$. Consequently,

$$
\forall(i, j) \in I_{\text {contact }}, D_{i j}(\tilde{\mathbf{q}}) \geq D_{i j}(\mathbf{q})+s \mathbf{G}_{i j}(\mathbf{q}) \cdot \mathbf{z}=s \mathbf{G}_{i j}(\mathbf{q}) \cdot \mathbf{z} \geq 0
$$

Furthermore, if $(i, j) \notin I_{\text {contact }}$, then $s \leq \frac{D_{i j}(\mathbf{q})}{\sqrt{2}|\mathbf{z}|}$. Hence

$$
D_{i j}(\tilde{\mathbf{q}}) \geq D_{i j}(\mathbf{q})+s \mathbf{G}_{i j}(\mathbf{q}) \cdot \mathbf{z} \geq D_{i j}(\mathbf{q})-s \sqrt{2}|\mathbf{z}| \geq 0
$$

That is why $\tilde{\mathbf{q}} \in Q$ and $d_{Q}(\mathbf{q}+s \mathbf{w}) \leq|\mathbf{q}+s \mathbf{w}-\tilde{\mathbf{q}}|=s|\mathbf{v}|$. Yet $|\mathbf{q}+s \mathbf{w}-\mathbf{q}|=s|\mathbf{w}|>s|\mathbf{v}|$ because $|\mathbf{w}|^{2}=|\mathbf{v}|^{2}+|\mathbf{z}|^{2}$. Thus $\mathbf{q} \notin \mathrm{P}_{Q}(\mathbf{q}+s \mathbf{w})$, which leads to a contradiction. In conclusion, $\mathbf{z}=0$ and $\mathbf{w}=\mathbf{v} \in \mathcal{N}_{\mathbf{q}}=\sum \mathrm{N}\left(Q_{i j}, \mathbf{q}\right)$, which completes the proof of the proposition.

Now we want to show the uniform prox-regularity of $Q$. Since $Q$ does not satisfy the same smoothness properties as $Q_{i j}$, the results of differential geometry cannot be applied. By Theorem 2.14, if a set is the complement of a smooth convex set, then it is uniformly prox-regular. A natural question arises: Is the intersection of such sets (which is the case for $Q$ ) uniformly prox-regular with a constant depending only on the constants of prox-regularity of the smooth sets. From a general point of view, this is wrong as illustrated in Figure 5. Indeed, we have plotted in solid line the boundary of a set $S$ which is the intersection of two identical disks' complements. This set is uniformly prox-regular but its constant of prox-regularity (equal to the radius of the disk plotted in dashed line) tends to zero when the disks' centres move away from each other. In this situation, the scalar product between normal vectors $\mathbf{n}_{1}$ and $\mathbf{n}_{2}$ (see Fig. 6) tends to -1 . Thus, the constant of prox-regularity of $S$ is also dependent on the angle between vectors $\mathbf{n}_{1}$ and $\mathbf{n}_{2}$. We now come to the main result of this section: the uniform prox-regularity of $Q$. This result rests on an inverse triangle inequality between vectors $\mathbf{G}_{i j}(\mathbf{q})$, which is based on angle estimates. Let us point out that we do not claim optimality of the constant $\eta$ below. 


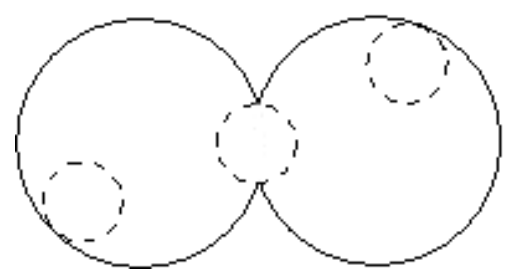

FIGURE 5. Vanishing of the constant of prox-regularity.

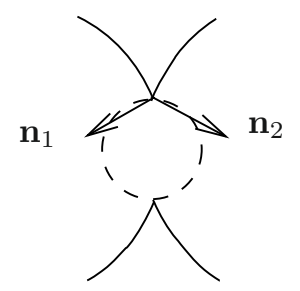

Figure 6 . Evolution of the angle between vectors $\mathbf{n}_{1}$ and $\mathbf{n}_{2}$.

Proposition 2.17. $Q$ is $\eta$-prox-regular with

$$
\eta \sim \frac{r \sqrt{2}}{2^{3 N}} \frac{1}{12^{3 N^{2}}}
$$

Proof. We want to prove ( $c f$. Def. 2.5) that there exists $\eta>0$ such that for all $\mathbf{q} \in Q$ and for all $\mathbf{v} \in \mathrm{N}(Q, \mathbf{q})$,

$$
\mathbf{v} \cdot(\tilde{\mathbf{q}}-\mathbf{q}) \leq \frac{|\mathbf{v}|}{2 \eta}|\tilde{\mathbf{q}}-\mathbf{q}|^{2}, \forall \tilde{\mathbf{q}} \in Q
$$

By Proposition 2.15, for all $\mathbf{q} \in Q_{i j}$ and all $\mathbf{w} \in \mathrm{N}\left(Q_{i j}, \mathbf{q}\right)$, we have

$$
\mathbf{w} \cdot(\tilde{\mathbf{q}}-\mathbf{q}) \leq \frac{|\mathbf{w}|}{2 \eta_{0}}|\tilde{\mathbf{q}}-\mathbf{q}|^{2}, \forall \tilde{\mathbf{q}} \in Q_{i j} .
$$

Inegality (2.6) is obvious when $\mathbf{v}=0$. So we consider $\mathbf{q} \in \partial Q$ and $\mathbf{v} \in N(Q, \mathbf{q}) \backslash\{0\}$. By Proposition 2.16,

$$
\mathbf{v}=-\sum_{(i, j) \in I_{\text {contact }}} \alpha_{i j} \mathbf{G}_{i j}(\mathbf{q}), \alpha_{i j} \geq 0 .
$$

We recall that $Q \subset Q_{i j}$ so that by (2.7) we obtain

$$
\left(-\sum \alpha_{i j} \mathbf{G}_{i j}(\mathbf{q})\right) \cdot(\tilde{\mathbf{q}}-\mathbf{q}) \leq \sum \frac{\alpha_{i j}\left|\mathbf{G}_{i j}(\mathbf{q})\right|}{2 \eta_{0}}|\tilde{\mathbf{q}}-\mathbf{q}|^{2}, \forall \tilde{\mathbf{q}} \in Q .
$$

The sum concerns only couples $(i, j)$ belonging to $I_{\text {contact }}$ but for convenience, this point is omitted in the notation. As $\left|\mathbf{G}_{i j}(\mathbf{q})\right|=\sqrt{2}$, we get

$$
\mathbf{v} \cdot(\tilde{\mathbf{q}}-\mathbf{q}) \leq \frac{1}{\sqrt{2} \eta_{0}}\left(\sum \alpha_{i j}\right)|\tilde{\mathbf{q}}-\mathbf{q}|^{2}, \forall \tilde{\mathbf{q}} \in Q
$$


To check inequality (2.6), it suffices to find a constant $\eta>0$, independent from $\alpha_{i j}$ and from $\mathbf{q}$, satisfying

$$
\left(\sum \alpha_{i j}\right) \frac{1}{\sqrt{2} \eta_{0}} \leq \frac{1}{2 \eta}\left|\sum \alpha_{i j} \mathbf{G}_{i j}(\mathbf{q})\right|
$$

i.e. such that

$$
\left|\sum \alpha_{i j} \mathbf{G}_{i j}(\mathbf{q})\right| \geq \sqrt{2} \frac{\eta}{\eta_{0}}\left(\sum \alpha_{i j}\right)
$$

Finally, if we are able to exhibit $\gamma>0$ verifying

$$
\left|\sum \alpha_{i j} \mathbf{G}_{i j}(\mathbf{q})\right| \geq \frac{\sqrt{2}}{\gamma}\left(\sum \alpha_{i j}\right)
$$

then $Q$ will be $\eta$-prox-regular with

$$
\eta=\frac{\eta_{0}}{\gamma}=\frac{r \sqrt{2}}{\gamma}
$$

The problem takes the form of an inverse triangle inequality:

$$
\sum \alpha_{i j}\left|\mathbf{G}_{i j}(\mathbf{q})\right|=\sqrt{2} \sum \alpha_{i j} \leq \gamma\left|\sum \alpha_{i j} \mathbf{G}_{i j}(\mathbf{q})\right| .
$$

The required result will follow as soon as we prove the main proposition stated below.

Proposition 2.18 (inverse triangle inequality). There exists $\gamma>1$ such that for all $\mathbf{q} \in Q$,

$$
\sum_{(i, j) \in I_{\text {contact }}} \alpha_{i j}\left|\mathbf{G}_{i j}(\mathbf{q})\right| \leq \gamma\left|\sum_{(i, j) \in I_{\text {contact }}} \alpha_{i j} \mathbf{G}_{i j}(\mathbf{q})\right|,
$$

where

$$
I_{\text {contact }}=\left\{(i, j), i<j, D_{i j}(\mathbf{q})=0\right\} \text { and } \alpha_{i j} \text { are nonnegative reals. }
$$

Constant $\gamma$ can be fixed as follows

$$
\gamma=\left[\frac{1}{2}\left(1-\left(1+\left(\frac{1}{12^{2 N}}\right)\right)^{-1 / 2}\right)\right]^{-\frac{3 N}{2}} .
$$

Remark 2.19. Note the sign of coefficients $\alpha_{i j}$. From a general point of view, this inequality is obviously wrong if these coefficients are just assumed real. Indeed, for $N$ large enough, the cardinal of the set $I_{\text {contact }}$ could be strictly larger than $2 N$, which induces a relation between vectors $\mathbf{G}_{i j}(\mathbf{q})$ (see Fig. 9 for such a degenerate situation).

The following elementary lemma asserts an inverse triangle inequality for two vectors.

Lemma 2.20. Let $u_{1}$ and $u_{2}$ be two vectors of $\mathbb{R}^{2 N}$ satisfying $u_{1} \cdot u_{2}=\cos \theta\left|u_{1}\right|\left|u_{2}\right|$, with $\cos \theta>-1$. Then for all

$$
\nu \geq \nu_{\theta}:=\sqrt{\frac{2}{1+\cos \theta}}
$$

we have $\left|u_{1}\right|+\left|u_{2}\right| \leq \nu\left|u_{1}+u_{2}\right|$. 
Proof of the inverse triangle inequality. We propose here a method based on angle estimates with vectors $\mathbf{G}_{i j}(\mathbf{q})$ as pointed out in Figure 6. We use a recursive proof on the number of involved vectors. We are going to check that there exists $\delta>1$ such that for all subset $I \subset I_{\text {contact }}$ and for all $\alpha_{i j}>0$,

$$
\sum_{(i, j) \in I \subset I_{\text {contact }}} \alpha_{i j}\left|\mathbf{G}_{i j}(\mathbf{q})\right| \leq \delta^{|I|}\left|\sum_{(i, j) \in I \subset I_{\text {contact }}} \alpha_{i j} \mathbf{G}_{i j}(\mathbf{q})\right| .
$$

Initialization: Suppose that the cardinality of $I$ equals to 1 , in other words, $I=\{(i, j)\}$. So we clearly have for all $\alpha_{i j}>0$ and all $\delta>1$,

$$
\alpha_{i j}\left|\mathbf{G}_{i j}(\mathbf{q})\right|=\left|\alpha_{i j} \mathbf{G}_{i j}(\mathbf{q})\right| \leq \delta\left|\alpha_{i j} \mathbf{G}_{i j}(\mathbf{q})\right| .
$$

Recursion assumption: If $|J|=p$, then we have for all $\alpha_{i j}>0$

$$
\sum_{(i, j) \in J \subset I_{\text {contact }}} \alpha_{i j}\left|\mathbf{G}_{i j}(\mathbf{q})\right| \leq \delta^{p}\left|\sum_{(i, j) \in J \subset I_{\text {contact }}} \alpha_{i j} \mathbf{G}_{i j}(\mathbf{q})\right| .
$$

Take a subset $I \subset I_{\text {contact }}$ with $|I|=p+1$. For any

$$
\mathbf{w}=\sum_{(i, j) \in I} \alpha_{i j} \mathbf{G}_{i j}(\mathbf{q})
$$

with $\alpha_{i j}>0$, we choose $(k, l) \in I$ and define $J=I \backslash\{(k, l)\}$,

$$
\mathbf{w}_{1}=\sum_{(i, j) \in J} \alpha_{i j} \mathbf{G}_{i j}(\mathbf{q}) \text { and } \mathbf{w}_{2}=\alpha_{k l} \mathbf{G}_{k l}(\mathbf{q}) .
$$

We need the following lemma which will be later proved.

Lemma 2.21. If $\mathbf{w}_{1} \neq 0$, the following inequality holds

$$
\frac{\mathbf{w}_{1} \cdot \mathbf{w}_{2}}{\left|\mathbf{w}_{1}\right|\left|\mathbf{w}_{2}\right|} \geq-\kappa, \text { with } \kappa=\left(1+\left(\frac{1}{12}\right)^{2 N}\right)^{-1 / 2} .
$$

Consequently, if $\mathbf{w}_{1} \neq 0$, from Lemma 2.20, we deduce $\left|\mathbf{w}_{1}\right|+\left|\mathbf{w}_{2}\right| \leq \sqrt{\frac{2}{1-\kappa}}\left|\mathbf{w}_{1}+\mathbf{w}_{2}\right|$ (this inequality obviously holds for $\left.\mathbf{w}_{1}=0\right)$. By denoting $\delta=\sqrt{\frac{2}{1-\kappa}}>1$, we get

$$
\left|\mathbf{w}_{1}\right|+\left|\mathbf{w}_{2}\right| \leq \delta|\mathbf{w}|
$$

Applying recursion assumption (2.9) and (2.10), we obtain

$$
\sum_{(i, j) \in I} \alpha_{i j}\left|\mathbf{G}_{i j}(\mathbf{q})\right| \leq \alpha_{k l}\left|\mathbf{G}_{k l}(\mathbf{q})\right|+\delta^{p}\left|\mathbf{w}_{1}\right| \leq \delta^{p}\left(\left|\mathbf{w}_{2}\right|+\left|\mathbf{w}_{1}\right|\right) \leq \delta^{p+1}|\mathbf{w}|
$$

which ends the proof of (2.8) by recursion. As $\left|I_{\text {contact }}\right| \leq 3 N$, the inverse triangle inequality is checked with $\gamma=\delta^{3 N}$. 


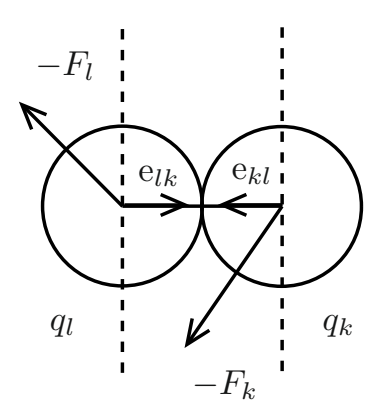

(a) Case 1

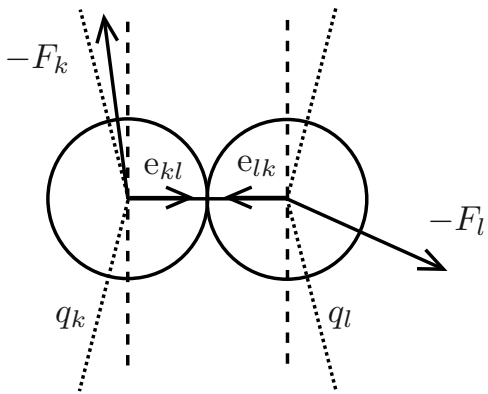

(b) Case 2a

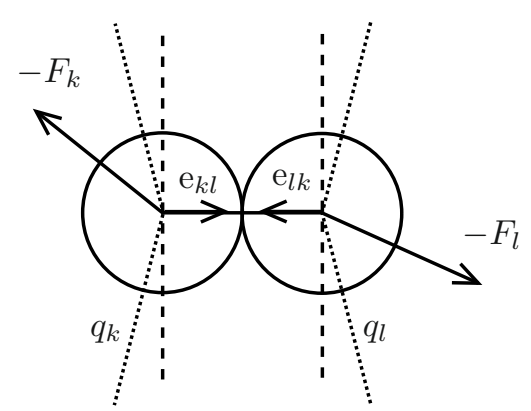

(c) Case $2 \mathrm{~b}$

FiguRE 7. Different relative positions of forces $F_{k}$ and $F_{l}$.

Proof of Lemma 2.21. It suffices to deal with $\mathbf{w}_{2}=\mathbf{G}_{k l}(\mathbf{q})$. By setting

$$
\beta_{i j}= \begin{cases}\alpha_{i j} & \text { if } i<j \\ \alpha_{j i} & \text { else }\end{cases}
$$

we have

$$
\mathbf{w}_{1}=\left(F_{1}, F_{2}, \ldots, F_{N}\right) \text { where } F_{p}=\sum \beta_{i p} \mathrm{e}_{i p} .
$$

Thus, $F_{k} \in \mathbb{R}^{2}$ can be interpreted as a pressure force exerted on the $k$-th person by its neighbours (different from the individual $l$ ). Similarly, $-F_{k}$ can be seen as a reaction force. We are looking for a lower bound of

$$
\Delta_{k l}:=\frac{\mathbf{w}_{1} \cdot \mathbf{w}_{2}}{\left|\mathbf{w}_{1}\right|\left|\mathbf{w}_{2}\right|}=\frac{-F_{k} \cdot \mathrm{e}_{k l}-F_{l} \cdot \mathrm{e}_{l k}}{\sqrt{2} \sqrt{\sum_{i=1}^{N}\left|F_{i}\right|^{2}}} .
$$

Case 1. $-F_{k} \cdot \mathrm{e}_{k l} \geq 0$ or $-F_{l} \cdot \mathrm{e}_{l k} \geq 0$.

Suppose that, for example (see Fig. 8a) $-F_{k} \cdot \mathrm{e}_{k l} \geq 0$. Using $\left|F_{l} \cdot \mathrm{e}_{l k}\right| \leq\left|F_{l}\right|$, we get

$$
\Delta_{k l} \geq \frac{-F_{l} \cdot \mathrm{e}_{l k}}{\sqrt{2} \sqrt{\sum\left|F_{i}\right|^{2}}} \geq \frac{-1}{\sqrt{2}} .
$$

In this case, $\kappa=2^{-1 / 2}$.

Case 2. $-F_{k} \cdot \mathrm{e}_{k l}<0$ and $-F_{l} \cdot \mathrm{e}_{l k}<0$.

Case 2a. $-F_{k} \cdot \mathrm{e}_{k l} \geq-\frac{1}{4}\left|F_{k}\right|$ or $-F_{l} \cdot \mathrm{e}_{l k} \geq-\frac{1}{4}\left|F_{l}\right|$.

Suppose that, for example (see Fig. $8 \mathrm{~b}$ ), $-F_{k} \cdot \mathrm{e}_{k l} \geq-\frac{1}{4}\left|F_{k}\right|$ It can be shown that

$$
-\frac{1}{4} \leq \frac{-F_{k} \cdot \mathrm{e}_{k l}}{\sqrt{\sum\left|F_{i}\right|^{2}}} \quad \text { and } \quad \frac{-F_{l} \cdot \mathrm{e}_{l k}}{\sqrt{\sum\left|F_{i}\right|^{2}}} \geq-1
$$

which yields

In this case $\kappa=5 /(4 \sqrt{2})$.

$$
\Delta_{k l} \geq \frac{1}{\sqrt{2}}\left(-\frac{1}{4}-1\right)=-\frac{5}{4 \sqrt{2}}>-1
$$


Case 2b. $-F_{k} \cdot \mathrm{e}_{k l}<-\frac{1}{4}\left|F_{k}\right|$ and $-F_{l} \cdot \mathrm{e}_{l k}<-\frac{1}{4}\left|F_{l}\right|$ (cf. Fig. 8c).

We need the following lemma.

Lemma 2.22. There exists $\tilde{k}$ and $\tilde{l}$ different from $k$ and $l$ verifying $\tilde{k} \neq \tilde{l}$ and

$$
\begin{aligned}
& \left|F_{\tilde{k}}\right| \geq \epsilon\left|F_{k}\right|, \\
& \left|F_{\tilde{l}}\right| \geq \epsilon\left|F_{l}\right|,
\end{aligned}
$$

with $\epsilon=1 / 12^{N}$.

We deduce that

$$
\sum\left|F_{i}\right|^{2} \geq\left|F_{k}\right|^{2}+\left|F_{l}\right|^{2}+\left|F_{\tilde{k}}\right|^{2}+\left|F_{\tilde{l}}\right|^{2} \geq\left(1+\epsilon^{2}\right)\left[\left|F_{k}\right|^{2}+\left|F_{l}\right|^{2}\right]
$$

Therefore

$$
\left|\Delta_{k l}\right| \leq \frac{1}{\sqrt{1+\epsilon^{2}}}\left(\frac{\left|F_{k}\right|+\left|F_{l}\right|}{\sqrt{2} \sqrt{\left|F_{k}\right|^{2}+\left|F_{l}\right|^{2}}}\right) \leq \frac{1}{\sqrt{1+\epsilon^{2}}} .
$$

In this case, $\kappa=\frac{1}{\sqrt{1+\epsilon^{2}}}$, which concludes the proof of Lemma 2.21 .

Proof of Lemma 2.22. We firstly consider

$$
-F_{k}=\sum_{i=1}^{V_{k}} \beta_{k j_{0, i}} \mathrm{e}_{k j_{0, i}}
$$

where $V_{k}$ is the number of neighbours of individual $k$ (individual $l$ excepted) $\left(V_{k} \leq 5\right)$. As a consequence,

$$
-F_{k} \cdot \mathrm{e}_{k l}=\sum_{i=1}^{V_{k}} \beta_{k j_{0, i}} \mathrm{e}_{k j_{0, i}} \cdot \mathrm{e}_{k l} .
$$

There exists $k_{1} \in\left\{j_{0,1}, j_{0,2}, \ldots, j_{0, V_{k}}\right\}\left(k_{1} \neq k, l\right)$ such that for all $i \in\left\{1, \ldots, V_{k}\right\} \beta_{k k_{1}} \mathrm{e}_{k k_{1}} \cdot \mathrm{e}_{k l} \leq \beta_{k j_{0, i}} \mathrm{e}_{k j_{0, i}} \cdot \mathrm{e}_{k l}$. It is obvious that

$$
\beta_{k k_{1}} \mathrm{e}_{k k_{1}} \cdot \mathrm{e}_{k l}<-\frac{1}{6} F_{k} \cdot \mathrm{e}_{k l} \leq-\frac{1}{24}\left|F_{k}\right| \text {. }
$$

In fact, individual $k_{1}$ is the neighbour who exerts the largest pressure force on person $k$. As illustrated in Figure 8, individual $k$ is between persons $l$ and $k_{1}$.

If $\left|F_{k_{1}}\right| \geq \frac{1}{48}\left|F_{k}\right|$, then we set $\tilde{k}=k_{1}$. Else $\left|F_{k_{1}}\right|<\frac{1}{48}\left|F_{k}\right|$, and we produce the same reasoning with

$$
-F_{k_{1}}=\beta_{k_{1} k} \mathrm{e}_{k_{1} k}+\sum_{i=1}^{V_{k_{1}}} \beta_{k_{1} j_{1, i}} \mathrm{e}_{k_{1} j_{1, i}}
$$

where $V_{k_{1}} \leq 5$. Thus,

$$
-F_{k_{1}} \cdot \mathrm{e}_{k l}=\beta_{k_{1} k} \mathrm{e}_{k_{1} k} \cdot \mathrm{e}_{k l}+\sum_{i=1}^{V_{k_{1}}} \beta_{k_{1} j_{1, i}} \mathrm{e}_{k_{1} j_{1, i}} \cdot \mathrm{e}_{k l} .
$$

Since $-\beta_{k_{1} k} \mathrm{e}_{k_{1} k} \cdot \mathrm{e}_{k l}<-\frac{1}{24}\left|F_{k}\right|$ and $-F_{k_{1}} \cdot \mathrm{e}_{k l} \leq\left|F_{k_{1}}\right|<\frac{1}{48}\left|F_{k}\right|$, we obtain

$$
\sum_{i=1}^{V_{k_{1}}} \beta_{k_{1} j_{1, i}} \mathrm{e}_{k_{1} j_{1, i}} \cdot \mathrm{e}_{k l}=-F_{k_{1}} \cdot \mathrm{e}_{k l}-\beta_{k_{1} k} \mathrm{e}_{k_{1} k} \cdot \mathrm{e}_{k l}<-\frac{1}{48}\left|F_{k}\right| .
$$



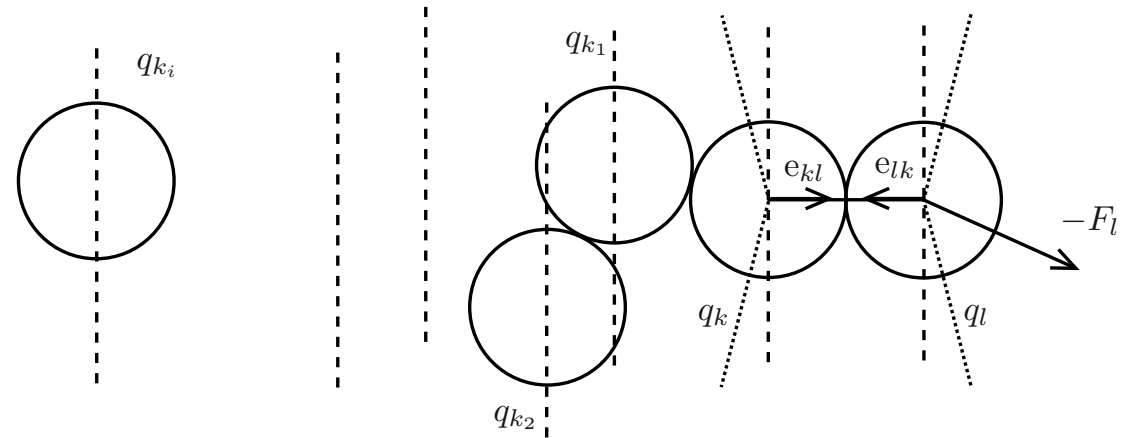

Figure 8. Construction of sequence $\left(k_{i}\right)$.

As previously, there exists $k_{2} \in\left\{j_{1,1}, j_{1,2}, \ldots, j_{1, V_{k_{1}}}\right\}\left(k_{2} \notin\left\{k, k_{1}\right\}\right)$, such that

$$
\beta_{k_{1} k_{2}} \mathrm{e}_{k_{1} k_{2}} \cdot \mathrm{e}_{k l}<-\frac{1}{48 \times 6}\left|F_{k}\right| .
$$

(Similarly, individual $k_{1}$ is between persons $k_{2}$ and $k$, see Fig. 8.)

If $\left|F_{k_{2}}\right| \geq \frac{1}{4}\left(\frac{1}{12}\right)^{2}\left|F_{k}\right|$, we set $\tilde{k}=k_{2}$. Else, we continue in defining a sequence $\left(k_{i}\right)$ (see Fig. 8) such that

$$
\left\{\begin{array}{l}
k_{0}=k \\
\left|F_{k_{i+1}}\right|<\frac{1}{4}\left(\frac{1}{12}\right)^{i+1}\left|F_{k}\right| \\
\beta_{k_{i} k_{i+1}} \mathrm{e}_{k_{i} k_{i+1}} \cdot \mathrm{e}_{k l}<-\frac{1}{4}\left(\frac{1}{12}\right)^{i} \frac{1}{6}\left|F_{k}\right| .
\end{array}\right.
$$

It can be shown that $k_{i+1} \notin\left\{k_{0}, k_{1}, \ldots, k_{i}\right\}$. This construction ends at most in $N-2$ steps:

$$
\exists m<N-1 \text { satisfying }\left|F_{k_{m}}\right| \geq \frac{1}{4}\left(\frac{1}{12}\right)^{m}\left|F_{k}\right|
$$

Finally we set

$$
\tilde{k}=k_{m} .
$$

Analogously, we deal with $F_{l}$, by constructing a sequence $\left(l_{i}\right)$ verifying similar properties. We can check that $\tilde{k} \neq \tilde{l}$ in proving that

$$
\left\{k_{0}, k_{1}, \ldots, k_{m}\right\} \cap\left\{l_{0}, l_{1}, \ldots, l_{p}\right\}=\emptyset .
$$

The proof of Lemma 2.22 is achieved by taking $\epsilon=1 / 12^{N}$.

\section{NUMERICAL SCHEME}

\subsection{Time-discretization scheme}

We present in this section a numerical scheme to approximate the solution to (2.4). The numerical scheme we propose is based on a first order expansion of the constraints expressed in terms of velocities. The time 
interval is denoted by $[0, T]$. Let $p \in \mathbb{N}^{\star}, h=T / p$ be the time step and $t^{n}=n h$ be the computational times. We denote by $\mathbf{q}^{n}$ the approximation of $\mathbf{q}\left(t^{n}\right)$. The next configuration is obtained as

$$
\mathbf{q}^{n+1}=\mathbf{q}^{n}+h \mathbf{u}^{n}
$$

where

$$
\begin{gathered}
\mathbf{u}^{n}=\mathrm{P}_{\mathcal{C}_{\mathbf{q}^{n}}^{h}}\left(\mathbf{U}\left(\mathbf{q}^{n}\right)\right) \text { with } \\
\mathcal{C}_{\mathbf{q}}^{h}=\left\{\mathbf{v} \in \mathbb{R}^{2 N}, D_{i j}(\mathbf{q})+h \mathbf{G}_{i j}(\mathbf{q}) \cdot \mathbf{v} \geq 0 \quad \forall i<j\right\} .
\end{gathered}
$$

The scheme can be also interpreted in the following way. Let us introduce the set

$$
\tilde{Q}(\mathbf{q})=\left\{\tilde{\mathbf{q}} \in \mathbb{R}^{2 N}, D_{i j}(\mathbf{q})+\mathbf{G}_{i j}(\mathbf{q}) \cdot(\tilde{\mathbf{q}}-\mathbf{q}) \geq 0 \quad \forall i<j\right\},
$$

which can be seen as an inner convex approximation of $Q$ with respect to q. Note that $\tilde{Q}(\mathbf{q})$ is defined in such a way that $Q$ is the union of all sets $\tilde{Q}(\mathbf{q}), \mathbf{q} \in Q$. The scheme can be expressed in terms of position:

$$
\mathbf{q}^{n+1}=\mathrm{P}_{\tilde{Q}\left(\mathbf{q}^{n}\right)}\left(\mathbf{q}^{n}+h \mathbf{U}\left(\mathbf{q}^{n}\right)\right) .
$$

In this form it appears as a prediction-correction algorithm: predicted position vector $\mathbf{q}^{n}+h \mathbf{U}\left(\mathbf{q}^{n}\right)$, that may not be admissible, is projected onto the approximate set of feasible configurations.

Remark 3.1. It is straightforward to check that

$$
\frac{\mathbf{q}^{n+1}-\mathbf{q}^{n}}{h}+\mathrm{N}\left(\tilde{Q}\left(\mathbf{q}^{n}\right), \mathbf{q}^{n+1}\right) \ni \mathbf{U}\left(\mathbf{q}^{n}\right),
$$

so that the scheme can also be seen as a semi-implicit discretization of (2.4), where $\mathrm{N}\left(\tilde{Q}\left(\mathbf{q}^{n}\right), \mathbf{q}^{n+1}\right)$ approximates $\mathrm{N}\left(Q, \mathbf{q}^{n}\right)$.

Convergence of this scheme shall be proven in a forthcoming paper [47].

\subsection{Numerical solutions}

In the model, the discrete actual velocity $\mathbf{u}^{n}$ is the projection of the spontaneous velocity onto the approximated set of feasible velocities. We propose here to solve this projection by a Uzawa algorithm (note that many other algorithms could be used to perform this quadratic minimization under affine unilateral constraints). For convenience, explicit dependence of vectors and matrices upon the current configuration is omitted (e.g. $\mathbf{U}$ stands for $\mathbf{U}\left(\mathbf{q}^{n}\right), D_{i j}$ for $D_{i j}\left(\mathbf{q}^{n}\right)$, etc.). The actual velocity $\mathbf{u}$ solves the following minimization problem under constraints

$$
\mathbf{u}=\underset{\mathbf{v} \in \mathcal{C}_{\mathbf{q}}^{h}}{\operatorname{argmin}}|\mathbf{v}-\mathbf{U}|^{2} .
$$

Uzawa algorithm is based on a reformulation of this minimization problem in a saddle-point form. We introduce the associated Lagrangian

$$
L(\mathbf{v}, \boldsymbol{\mu})=\frac{1}{2}|\mathbf{v}-\mathbf{U}|^{2}-\sum_{1 \leq i<j \leq N} \mu_{i j}\left(D_{i j}+h \mathbf{G}_{i j} \cdot \mathbf{v}\right),
$$

and the following linear mapping

$$
\begin{aligned}
B: & \mathbb{R}^{2 N} \\
\mathbf{v} & \mapsto-\mathbb{R}^{\frac{N(N-1)}{2}} \\
& \mapsto\left(\mathbf{G}_{i j} \cdot \mathbf{v}\right)_{i<j} .
\end{aligned}
$$




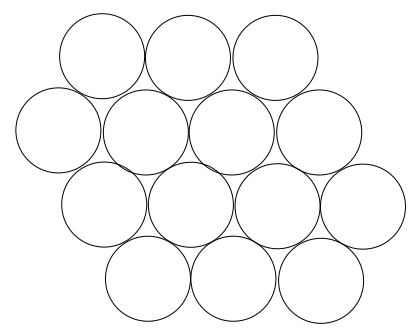

Figure 9. A case of non-uniqueness for Kuhn-Tucker multipliers.

With these notations, the set $\mathcal{C}_{\mathbf{q}}^{h}$ can be written:

$$
\begin{aligned}
\mathcal{C}_{\mathbf{q}}^{h} & =\left\{\mathbf{v} \in \mathbb{R}^{2 N}, \forall \boldsymbol{\mu} \in\left(\mathbb{R}^{+}\right)^{\frac{N(N-1)}{2}},-\sum_{1 \leq i<j \leq N} \mu_{i j}\left(D_{i j}+h \mathbf{G}_{i j} \cdot \mathbf{v}\right) \leq 0\right\} \\
& =\left\{\mathbf{v} \in \mathbb{R}^{2 N}, \forall \boldsymbol{\mu} \in\left(\mathbb{R}^{+}\right)^{\frac{N(N-1)}{2}}, \boldsymbol{\mu} \cdot(B \mathbf{v}-D) \leq 0\right\}
\end{aligned}
$$

where $D=D(\mathbf{q}) \in \mathbb{R}^{N(N-1) / 2}$ is the vector of distances. The existence of a saddle-point

$$
(\mathbf{u}, \boldsymbol{\lambda}) \in \mathbb{R}^{2 N} \times\left(\mathbb{R}^{+}\right)^{\frac{N(N-1)}{2}}
$$

for this problem is well-known (see e.g. [10]) and it is characterized by the next system:

$$
\left\{\begin{array}{l}
\mathbf{u}+{ }^{t} B \boldsymbol{\lambda}=\mathbf{U} \\
\boldsymbol{\mu} \cdot(B \mathbf{u}-D) \leq 0, \forall \boldsymbol{\mu} \geq 0 \\
\boldsymbol{\lambda} \cdot(B \mathbf{u}-D)=0
\end{array}\right.
$$

To compute this saddle-point, Uzawa algorithm is used and produces two sequences $\left(\mathbf{v}^{k}\right) \in\left(\mathbb{R}^{2 N}\right)^{\mathbb{N}}$ and $\left(\boldsymbol{\mu}^{k}\right) \in$ $\left(\left(\mathbb{R}^{+}\right)^{\frac{N(N-1)}{2}}\right)^{\mathbb{N}}$ according to

$$
\begin{aligned}
\boldsymbol{\mu}^{0} & =0 \\
\mathbf{v}^{k+1} & =\mathbf{U}-{ }^{t} B \boldsymbol{\mu}^{k} \\
\boldsymbol{\mu}^{k+1} & =\Pi_{+}\left(\boldsymbol{\mu}^{k}+\rho\left[B \mathbf{v}^{k+1}-D\right]\right)
\end{aligned}
$$

where $\Pi_{+}$is the Euclidean projection onto the cone of vectors with nonnegative components (a simple cut-off in practice), and $\rho>0$ is a fixed parameter. The Kuhn-Tuckers multipliers are initialized to zero and the algorithm (3.2), (3.3) is executed until for all $(i, j), D_{i j}\left(\mathbf{v}^{k+1}\right) \geq-\varepsilon$, where $\varepsilon>0$ is chosen small enough with respect to the radii.

The algorithm can be shown to converge as soon as $0<\rho<2 /\|B\|^{2}$ (see [10]). More precisely, the sequence $\left(\mathbf{v}^{k}\right)$ converges to $\mathbf{u}$ and it can be shown that the sequence $\left(\boldsymbol{\mu}^{k}\right)$ tends to some $\bar{\lambda} \in\left(\mathbb{R}^{+}\right)^{\frac{N(N-1)}{2}}$ such that $(\mathbf{u}, \overline{\boldsymbol{\lambda}})$ is a saddle-point of $L$. Notice that in general, the Kuhn-Tucker multiplier $\boldsymbol{\lambda}$ is not unique as illustrated in Figure 9. In this case, the configuration of 14 people shows 29 contacts, consequently matrix ${ }^{t} B$ is not injective.

Remark 3.2 (link between local prox-regularity and speed of convergence for Uzawa algorithm). We denote by $G$ the matrix whose columns are vectors $\mathbf{G}_{i j}$, where $(i, j) \in I_{\text {contact }}$ (defined by (2.5)), and we introduce 
$A={ }^{t} G G$. The size of this square matrix is equal to $n_{\text {contact }}$ which is the cardinal of $I_{\text {contact }}$. By the inverse triangle inequality (see Prop. 2.18), there exists a constant $\gamma$ such that for all $\boldsymbol{\lambda} \in\left(\mathbb{R}^{+}\right)^{n_{\text {contact }}}$ satisfying $|\boldsymbol{\lambda}|_{1}=1$, we have

$$
\left|\sum \lambda_{i j} \mathbf{G}_{i j}\right|^{2}={ }^{t} \boldsymbol{\lambda}^{t} G G \boldsymbol{\lambda}={ }^{t} \boldsymbol{\lambda} A \boldsymbol{\lambda} \geq \frac{2}{\gamma^{2}} .
$$

We define, for $\mathbf{q} \in Q$, a local parameter $\gamma_{\mathbf{q}}$ satisfying

$$
\min _{\substack{\left.|\lambda|\right|_{1}=1 \\ \lambda \geq 0}}{ }^{t} \boldsymbol{\lambda} A \boldsymbol{\lambda}=\frac{2}{\gamma_{\mathbf{q}}^{2}}
$$

and $\eta_{\mathbf{q}}=r \sqrt{2} / \gamma_{\mathbf{q}}$. Let us show that parameter $\eta_{\mathbf{q}}$ (setting a lower bound of the local prox-regularity of $Q$ at point $\mathbf{q}$ ) and the condition number of matrix $A$ are closely related when $A$ is non-singular. By denoting $\eta_{\min }$ the smallest eigenvalue of $A$, it follows that

$$
\eta_{\min }=\min _{|\boldsymbol{\lambda}|_{2}=1}{ }^{t} \boldsymbol{\lambda} A \boldsymbol{\lambda}=\min _{|\boldsymbol{\lambda}|_{2} \geq 1}{ }^{t} \boldsymbol{\lambda} A \boldsymbol{\lambda} \leq \min _{\substack{|\boldsymbol{\lambda}|_{2} \geq 1 \\ \boldsymbol{\lambda} \geq 0}}{ }^{t} \boldsymbol{\lambda} A \boldsymbol{\lambda} .
$$

Since for all $\boldsymbol{\lambda},|\boldsymbol{\lambda}|_{1} \leq \sqrt{n_{\text {contact }}}|\boldsymbol{\lambda}|_{2}$, we have

$$
\min _{\substack{|\lambda|_{2} \geq 1 \\ \lambda \geq 0}}{ }^{t} \boldsymbol{\lambda} A \boldsymbol{\lambda} \leq \min _{\substack{|\boldsymbol{\lambda}|_{1} \geq \sqrt{\text { contact }} \\ \boldsymbol{\lambda} \geq 0}}{ }^{t} \boldsymbol{\lambda} A \boldsymbol{\lambda}=n_{\text {contact }} \min _{\substack{|\lambda|_{1} \geq 1 \\ \lambda \geq 0}}{ }^{t} \boldsymbol{\lambda} A \boldsymbol{\lambda}
$$

Finally,

Thus

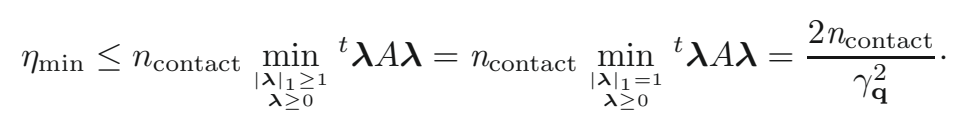

Furthermore, the condition number of matrix $A$ equals to

$$
\eta_{\min } \leq \frac{6 N}{\gamma_{\mathbf{q}}^{2}}
$$

$$
\operatorname{cond}_{2}(A)=\|A\|_{2}\left\|A^{-1}\right\|_{2}=\frac{\eta_{\max }}{\eta_{\min }} .
$$

Since $\left|\mathbf{G}_{i j}(\mathbf{q})\right|=\sqrt{2}$, we obtain $\|A\|_{2}=\eta_{\max } \geq 2$, hence

$$
\operatorname{cond}_{2}(A) \geq \frac{2}{\eta_{\min }} \geq \frac{2 \gamma_{\mathbf{q}}^{2}}{6 N} \geq \frac{4 r^{2}}{6 \eta_{\mathbf{q}}^{2} N}
$$

which quantifies how the condition number of $A$ varies with $\eta_{\mathbf{q}}$. Since the matrix appearing in Uzawa algorithm is $A={ }^{t} G G$, we expect that this algorithm converges less quickly for configurations with low local prox-regularity. In numerical simulations, we noticed indeed that solving the saddle-point problem requires more iterations in case of a jam.

\section{Numerical RESUlts}

In order to illustrate the contact model, we propose here an example of spontaneous velocity. The choice of the spontaneous velocity is important because this velocity reflects pedestrian behaviour. A lot of choices are obviously possible. The spontaneous velocity of an individual has to take into account obstacles in the room and specify how he wants to get around them. So this velocity depends on the room's geometry but it can be made dependent on other people positions too. Indeed, it is possible here to integrate individual strategies (deceleration or jam's avoiding). We refer the reader to $[35,36,46]$ for other examples of spontaneous velocity. 

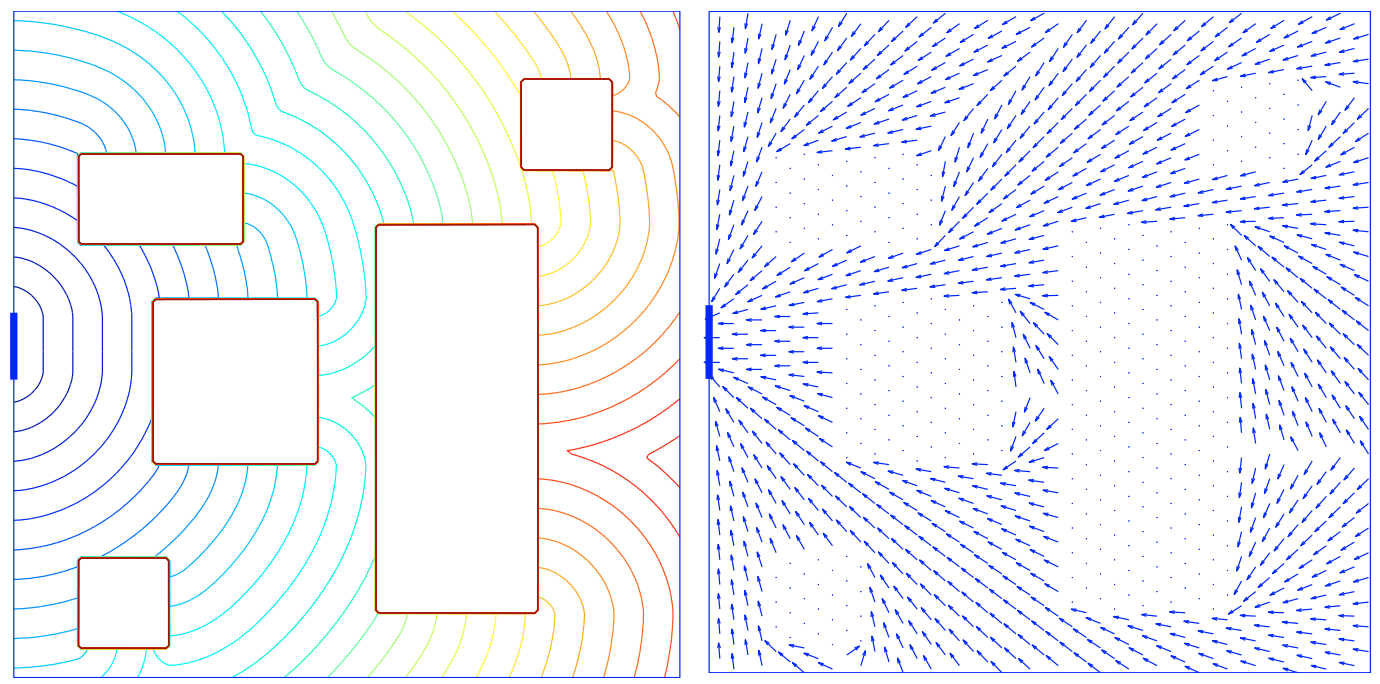

Figure 10. Contour levels of the geodesic distance $\mathcal{D}$ and velocity field $\mathbf{U}_{0}$.

Here we restrict ourselves to simple behavourial model: people tend to optimize their own path, regardless of others.

\section{An example of spontaneous velocity}

We consider here the simplest choice for the spontaneous velocity. All the individuals have the same behaviour: they want to reach the exit by following the shortest path avoiding obstacles. Then, the spontaneous velocity's expression can be specified:

$$
\mathbf{U}(\mathrm{q})=\left(\mathbf{U}_{0}\left(\mathrm{q}_{1}\right), \ldots, \mathbf{U}_{0}\left(\mathrm{q}_{N}\right)\right) \text { with } \mathbf{U}_{0}(\mathbf{x})=-\mathrm{s} \nabla \mathcal{D}(\mathbf{x})
$$

where $\mathcal{D}(\mathbf{x})$ represents the geodesic distance between the position $\mathbf{x}$ and the nearest exit and $\mathrm{s}>0$ denotes the speed. In order to compute $\mathcal{D}$, we have used the Fast Marching Method introduced by Kimmel and Sethian in [29]. In this method, the value of $\mathcal{D}$ is computed at each point of a grid. The value at the exit's nodes is set to zero. Then, the values of the distance at the other points is computed step by step so that a discrete version of $|\nabla \mathcal{D}|=1$ is satisfied. Moreover, the distance at the nodes situated in the obstacles is fixed to a large value, which prevents the shortest path from going across them. In Figure 10, we have considered a room with five obstacles and the exit is situated to the left. We note that by following the built velocity field, people are going to avoid obstacles. Our aim is to simulate evacuation of any building consisting of several floors. We have chosen an object oriented programming method and we have implemented this Fast Marching Method in a $\mathrm{C}++$ code. Let us detail this code. On each floor, the spontaneous velocity is directed by the shortest path avoiding obstacles to the nearest exit or stairwell. In the stairs, people just want to go down. We have integrated this spontaneous velocity in the $\mathrm{C}++$ code SCoPI: Simulations of Collections of Interacting Particles developed by Lefebvre (see $[32,33]$ ). This code allows us to compute the actual velocity as the projection of the spontaneous velocity as described in Section 3.

Remark 4.1. Notice that the velocity field produced by this strategy is not continuous as soon as the room is not convex, which rules out Theorem 2.10. This lack of regularity is not important in practical applications: the places at which it occurs (in particular upstream obstacles) are emptied after a few moments. The main consequence is the discontinuity of the future configurations with respect to initial data, which is not surprising from a modelling standpoint. 


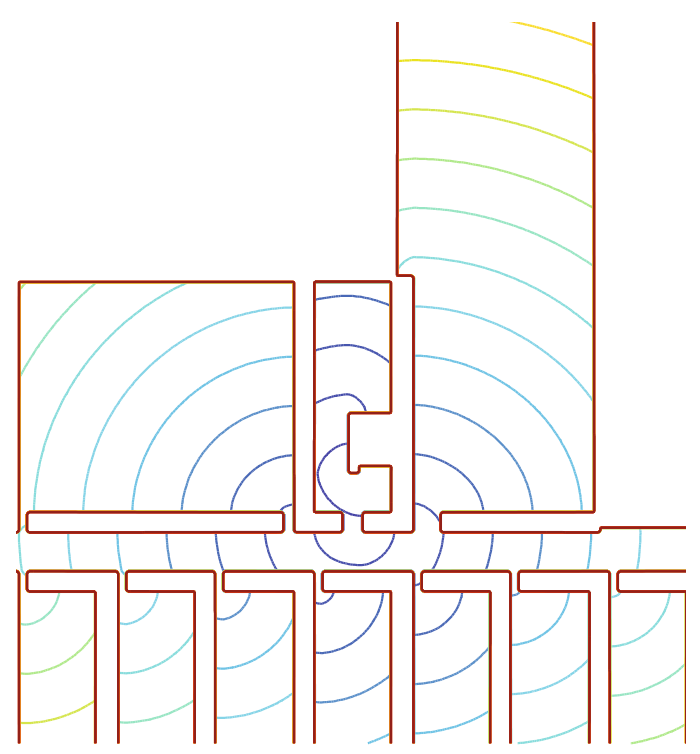

Figure 11. Geometry and isovalues for the geodesic distance.

We propose to illustrate the behaviour of the algorithm in two situations. The first one corresponds to a many-individual evacuation from a square room through a single exit, the second one illustrate the capability of the approach to handle complicated geometries. For these two experiments, it will be noticed that the contacts between the individuals and the obstacles have to be handled (as the contacts between people). Even if an individual want to avoid an obstacle, he can be pushed on it by people behind them.

\section{Simple evacuation}

We consider the situation of 1000 people which are randomly distributed over a square room. The spontaneous velocity field corresponds to straight pathlines towards the exit at constant speed. As the field has a negative divergence, it tends to increase the local density, so that congestion is rapidly reached in the neighbourhood of the exit, and the congestion front propagated upstream as long as it is feeded by incoming people. In Figure 12, we represented the current configuration and the corresponding network of interaction pressures: for any couple of disks in contact, we represent the segment between centers, having its color (from white to black) depend upon the (positive) Kuhn-Tucker multiplier which handles the corresponding constraint. We recover the apparition of arches upstream the exit. The Kuhn-Tucker multipliers $\lambda_{i j}$ quantify the way $\mathbf{U}$, the spontaneous velocity field, does not fit the constraints, and as such they can be interpreted in terms of pressures undergone by individuals. Although it would be presumptuous at this stage to assimilate $\lambda_{i j}$ to an actual measure of the discomfort experienced by persons $i$ and $j$, it is obvious that high values for those Kuhn-Tucker multipliers can be expected on zones where people are likely to be crushed.

\section{Complex geometry}

In the second example we consider the evacuation of a floor through exit stairs. A zoom on the geometry near the exit (together with the isovalues of the geodesic distance function, on which the spontaneous velocity is built) is represented in Figure 11. Figure 13 corresponds to snapshots at times $0 \mathrm{~s}, 5 \mathrm{~s}, 11 \mathrm{~s}, 16 \mathrm{~s}, 41 \mathrm{~s}$ and $75 \mathrm{~s}$. Disks are colored according to their initial geodesic distance to the exit. Note that initial ordering is not preserved during the evacuation. Notice also how a jam forms between snapshots 2 and 3 in the room located 

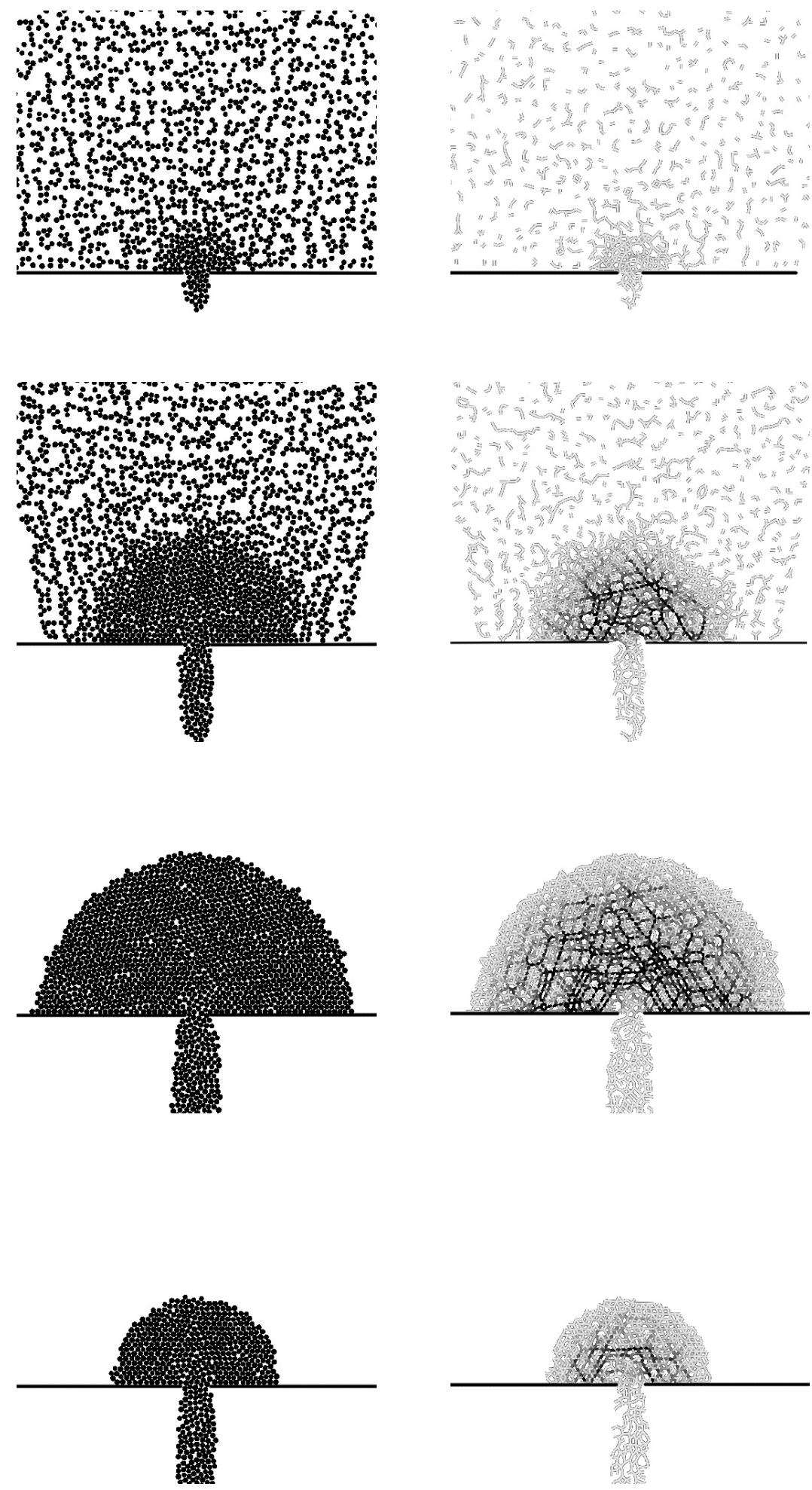

FIGURE 12. Configurations and corresponding networks of interaction pressures: observation of arches. 

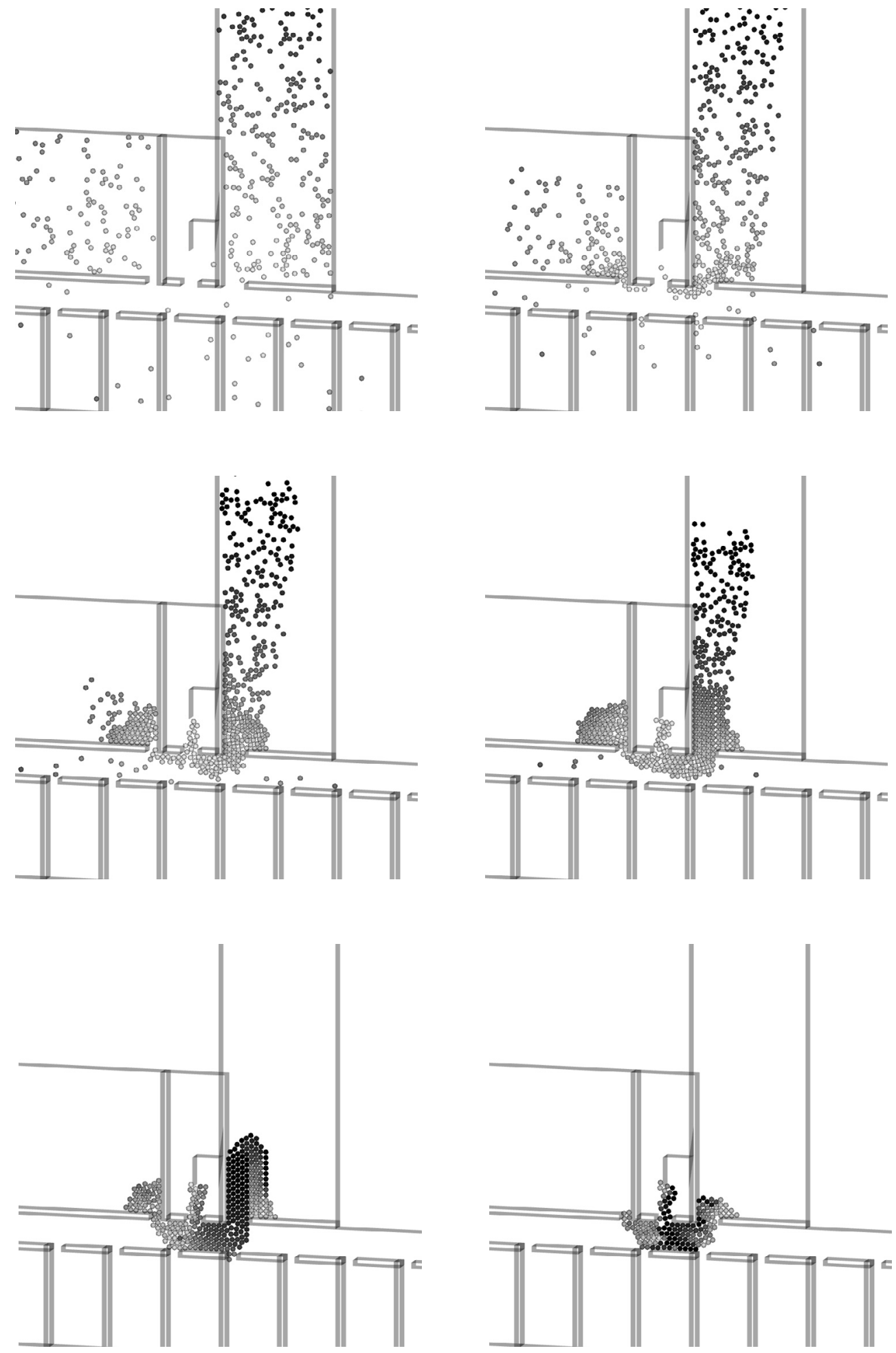

FiguRE 13. Formation of a jam during an evacuation. 
on the left hand side. This jam decreases significantly the rate at which people exit the room, but it disappears eventually. The final evacuation time is $109 \mathrm{~s}$, to be compared to $48 \mathrm{~s}$ which corresponds to the evacuation time without congestion.

\section{REFERENCES}

[1] N. Bellomo and C. Dogbe, On the modelling crowd dynamics from scalling to hyperbolic macroscopic models. Math. Mod. Meth. Appl. Sci. 18 (2008) 1317-1345.

[2] F. Bernicot and J. Venel, Existence of sweeping process in Banach spaces under directional prox-regularity. J. Convex Anal. 17 (2010) 451-484.

[3] V. Blue and J.L. Adler, Cellular automata microsimulation for modeling bi-directional pedestrian walkways. Transp. Res. B 35 (2001) 293-312.

[4] A. Borgers and H. Timmermans, City centre entry points, store location patterns and pedestrian route choice behaviour: A microlevel simulation model. Socio-Econ. Plann. Sci. 20 (1986) 25-31.

[5] A. Borgers and H. Timmermans, A model of pedestrian route choice and demand for retail facilities within inner-cityshopping areas. Geogr. Anal. 18 (1986) 115-128.

[6] M. Bounkhel and L. Thibault, On various notions of regularity of sets in nonsmooth analysis. Nonlinear Convex Anal. 48 (2002) 223-246.

[7] H. Brezis, Opérateurs Maximaux Monotones et Semi-groupes de Contractions dans les Espaces de Hilbert. AM, North Holland (1973).

[8] C. Burstedde, K. Klauck, A. Schadschneider and J. Zittartz, Simulation of pedestrian dynamics using a two-dimensional cellular automaton. Physica A 295 (2001) 507-525.

[9] G. Buttazzo, C. Jimenez and E. Oudet, An optimization problem for mass transportation with congested dynamics. SIAM J. Control Optim. 48 (2009) 1961-1976.

[10] P.G. Ciarlet, Introduction à l'analyse numérique matricielle et à l'optimisation. Masson, Paris (1990).

[11] F.H. Clarke, R.J. Stern and P.R. Wolenski, Proximal smoothness and the lower-c ${ }^{2}$ property. J. Convex Anal. 2 (1995) $117-144$.

[12] F.H. Clarke, Y.S. Ledyaev, R.J. Stern and P.R. Wolenski, Nonsmooth Analysis and Control Theory. Springer-Verlag, New York, Inc. (1998).

[13] W. Daamen, Modelling passenger flows in public transport facilities. Ph.D. Thesis, Technische Universiteit Delft, The Netherlands (2004).

[14] J.A. Delgado, Blaschke's theorem for convex hypersurfaces. J. Diff. Geom. 14 (1979) 489-496.

[15] C. Dogbé, On the numerical solutions of second order macroscopic models of pedestrian flows. Comput. Math. Appl. 56 (2008) $1884-1898$.

[16] J.F. Edmond and L. Thibault, Relaxation of an optimal control problem involving a perturbed sweeping process. Math. Program., Ser. B 104 (2005) 347-373.

[17] J.F. Edmond and L. Thibault, BV solutions of nonconvex sweeping process differential inclusion with perturbation. J. Diff. Equ. 226 (2006) 135-179.

[18] J.J. Fruin, Design for pedestrians: A level-of-service concept. Highway Research Record 355 (1971) 1-15.

[19] S. Gwynne, E.R. Galea, P.J. Lawrence and L. Filippidis, Modelling occupant interaction with fire conditions using the buildingEXODUS evacuation model. Fire Saf. J. 36 (2001) 327-357.

[20] D. Helbing, A fluid-dynamic model for the movement of pedestrians. Complex Syst. 6 (1992) 391-415.

[21] D. Helbing and P. Molnár, Social force model for pedestrians dynamics. Phys. Rev. E 51 (1995) 4282-4286.

[22] D. Helbing, I.J. Farkas and T. Vicsek, Simulating dynamical features of escape panic. Nature 407 (2000) 487.

[23] L.F. Henderson, The stastitics of crowd fluids. Nature 229 (1971) 381-383.

[24] S.P. Hoogendoorn and P.H.L. Bovy, Gas-kinetic modeling and simulation of pedestrian flows. Transp. Res. Rec. 1710 (2000) $28-36$.

[25] S.P. Hoogendoorn and P.H.L. Bovy, Pedestrian route-choice and activity scheduling theory and models. Transp. Res. B 38 (2004) 169-190

[26] S.P. Hoogendoorn and P.H.L. Bovy, Dynamic user-optimal assignment in continuous time and space. Transp. Res. B 38 (2004) $571-592$.

[27] R. Hughes, The flow of large crowds of pedestrians. Math. Comput. Simul. 53 (2000) 367-370.

[28] R. Hughes, A continuum theory for the flow of pedestrians. Transp. Res. B 36 (2002) 507-535.

[29] R. Kimmel and J. Sethian, Fast marching methods for computing distance maps and shortest paths. Technical Report 669, CPAM, Univ. of California, Berkeley (1996).

[30] A. Kirchner and A. Schadschneider, Simulation of evacuation processes using a bionics-inspired cellular automaton model for pedestrians dynamics. Physica A 312 (2002) 260-276.

[31] H. Klüpfel and T. Meyer-König, Characteristics of the pedgo software for crowd movement and egress simulation, in Pedestrian and Evacuation Dynamics 2003, E. Galea Ed., University of Greenwich, CMS Press, London (2003) 331-340. 
[32] A. Lefebvre, Modélisation numérique d'écoulements fluide/particules, Prise en compte des forces de lubrification. Ph.D. Thesis, Université Paris-Sud XI, Faculté des sciences d'Orsay, France (2007).

[33] A. Lefebvre, Numerical simulations of gluey particles. ESAIM: M2AN 43 (2009) 53-80.

[34] G.G. Løvås, Modelling and simulation of pedestrian traffic flow. Transp. Res. B 28 (1994) 429-443.

[35] B. Maury and J. Venel, Un modèle de mouvement de foule. ESAIM: Proc. 18 (2007) 143-152.

[36] B. Maury and J. Venel, Handling of contacts on crowd motion simulations, in Trafic and Granular Flow '07, Springer (2009) $171-180$.

[37] B. Maury, A. Roudneff-Chupin and F. Santambrogio, A macroscopic crowd motion model of gradient flow type. Math. Mod. Meth. Appl. Sci. (to appear). Available at http://cvgmt.sns.it/papers/maurousan09/.

[38] J.J. Moreau, Décomposition orthogonale d'un espace hilbertien selon deux cônes mutuellement polaires. C. R. Acad. Sci., Ser. I 255 (1962) 238-240.

[39] J.J. Moreau, Evolution problem associated with a moving convex set in a Hilbert space. J. Diff. Equ. 26 (1977) $347-374$.

[40] K. Nagel, From particle hopping models to traffic flow theory. Transp. Res. Rec. 1644 (1998) 1-9.

[41] P.D. Navin and R.J. Wheeler, Pedestrian flow characteristics. Traffic Engineering 39 (1969) 31-36.

[42] A. Schadschneider, Cellular automaton approach to pedestrian dynamics-theory, in Pedestrian and Evacuation Dynamics, M. Schreckenberg and S.D. Sharma Eds., Springer, Berlin (2001) 75-85.

[43] A. Schadschneider, A. Kirchner and K. Nishinari, From ant trails to pedestrian dynamics. Appl. Bionics G Biomechanics 1 (2003) 11-19.

[44] G.K. Still, New computer system can predict human behavior response to building fires. Fire 84 (1993) 40-41.

[45] J. Venel, Modélisation mathématique et numérique des mouvements de foule. Ph.D. Thesis, Université Paris-Sud XI, France, available at: http://tel.archives-ouvertes.fr/tel-00346035/fr (2008).

[46] J. Venel, Integrating strategies in numerical modelling of crowd motion, in Pedestrian and Evacuation Dynamics '08, W.W.F. Klingsch, C. Rogsch, A. Schadschneider and M. Schreckenberg Eds., Springer, Berlin Heidelberg (2010) 641-646.

[47] J. Venel, Numerical scheme for a whole class of sweeping process. Available at: http://arxiv.org/abs/0904.2694v2 (submitted).

[48] U. Weidmann, Transporttechnik der fussgaenger. Technical Report 90, Schriftenreihe des Instituts für Verkehrsplanung, Transporttechnik, Strassen-und Eisenbahnbau, ETH Zürich, Switzerland (1993).

[49] S.J. Yuhaski and J.M. Macgregor Smith, Modelling circulation systems in buildings using state dependent queueing models. Queue. Syst. 4 (1989) 319-338. 